\title{
Histone H2A ubiquitination resulting from Brap loss of function connects multiple aging hallmarks and accelerates neurodegeneration
}

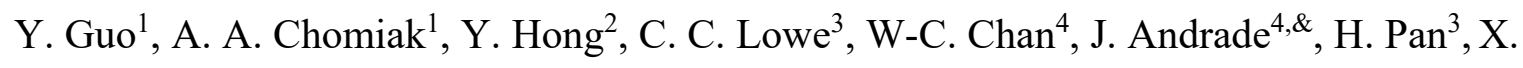

Zhou $^{5}$, E. Berezovski ${ }^{3}$, E. S. Monuki ${ }^{6}$, and Y. Feng ${ }^{1,3^{*}}$

1. Department of Neurology, Northwestern University Feinberg School of Medicine, 303 E.

Superior Street, Chicago, IL 60611, U.S.A

2. University of Turku, 20500 Turku, Finland

3. Department of Biochemistry and Molecular Biology, Uniformed Services University, 4301

Jones Bridge Road, Bethesda, MD 20814, U.S.A

4. Center for Research Informatics, University of Chicago, Chicago, IL 60637, U.S.A

5. Department of Medicine, Uniformed Services University, 4301 Jones Bridge Road, Bethesda, MD 20814, U.S.A

6. Department of Pathology \& Laboratory Medicine, University of California, Irvine, Irvine, CA 92697, U.S.A

\& Present address: Kite Pharma, Santa Monica, CA 90404, U.S.A

*To whom correspondence should be addressed:

Tel: (301) 295-9419

Email: yuanyi.feng@usuhs.edu 


\begin{abstract}
Aging is an intricate process that is characterized by multiple hallmarks including stem cell exhaustion, genome instability, epigenome alteration, impaired proteostasis, and cellular senescence. While each of these is detrimental at the cellular level, it remains unclear how they are interconnected to cause systemic organ deterioration. Here we show that abrogating Brap, a BRCA1 associated protein, results in cellular senescence with persistent DNA double-strand breaks and elevation of histone H2A mono- and poly-ubiquitination (H2Aub). The high H2Aub initiates proteasome-dependent histone proteolysis, leading to global epigenetic alteration, ubiquitinated protein accumulation, and senescence reinforcement. When these defects occur in mice carrying Brap deletions in cerebral cortical neural progenitors or postnatal neurons, they accelerate brain aging, induce neurodegeneration, and shorten lifespan. As we show H2Aub is also increased in human brain tissues of Alzheimer's disease, these data together suggest that chromatin aberrations mediated by $\mathrm{H} 2 \mathrm{Aub}$ act as a nexus of multiple aging hallmarks.
\end{abstract}




\section{INTRODUCTION}

Aging is a natural process that connects birth and death. The time-dependent decline in organ function during aging is the major risk factor for developing many diseases including neurodegenerative disorders (NDs). Yet, the etiology of aging is complex and multifactorial. It is associated with several cellular and molecular hallmarks, such as stem cell exhaustion, genome instability, epigenetic alteration, deregulated nutrient sensing, mitochondrial dysfunction, cellular senescence, and loss of proteostasis (Lopez-Otin et al., 2013). For neurodegeneration, additional hallmark defects in neuronal calcium homeostasis and neuronetwork activity, as well as increased glial cell activation and neuroinflammation are also essential contributors to the physical and functional deterioration of the brain (Mattson and Arumugam, 2018). Aging at the tissue level does not simply result from the dysfunction of any individual cellular or molecular process. To cause progressive and irreversible organ-wide degeneration, multiple aging promoting factors must act in concert. However, the mechanism underlying interconnections among various aging hallmarks is poorly understood, forming a major barrier to combating aging-associated diseases.

Among various aging hallmarks, genome instability poses a lifelong threat to all living cells and can result in increased somatic mutations that have been shown to accumulate with age in most organs (Lombard et al., 2005; Soares et al., 2014; White and Vijg, 2016). Besides a clear link to carcinogenesis, loss of genome stability has also been implicated in neurodegeneration (Bushman et al., 2015; Madabhushi et al., 2014; Mitra et al., 2019; Rulten and Caldecott, 2013; Shanbhag et al., 2019; Thadathil et al., 2019), and there has been long-established evidence for significant levels of DNA damage in non-dividing cells, such as neurons in the brain (Lodato et al., 2015; Lu et al., 2004; Rutten et al., 2007; Suberbielle et al., 2013). Recent whole genome 
sequencing analyses have further demonstrated that somatic mutations increase with age in both bulk tissues and single neurons of the human brain (Hoang et al., 2016; Lodato et al., 2018). Moreover, mutations of genes important for genome maintenance and DNA repair cause progeroid syndromes and often manifest with both premature aging and neurodegeneration (Choy and Watters, 2018; Coppede and Migliore, 2010; Kakigi et al., 1992; Weidenheim et al., 2009). Thus, genotoxicity is a universal insult that not only is concomitant with old age but also drives aging-associated diseases. Nevertheless, the mechanism by which neuronal DNA damage causes brain aging and neurodegeneration at the tissue level remains elusive.

A plausible mechanism for DNA damage to induce tissue-wide degeneration is via cellular senescence, a stress response that occurs when cells experience telomere erosion, chronic oncogenic stimulation, persistent DNA damage, oxidative and metabolic stresses, or mitochondrial dysfunction (Hayflick, 1965; Hernandez-Segura et al., 2018; McHugh and Gil, 2018; Munoz-Espin and Serrano, 2014; Rodier and Campisi, 2011). Although originally defined by a permanent replication arrest, cellular senescence is also characterized by a set of distinctive phenotypes, including activation of senescence-associated $\beta$-galactosidase (SA- $\beta$-gal), expression of a cyclin-dependent kinase inhibitor $(\mathrm{CKI}) \mathrm{p} 16^{\mathrm{Ink} 4 \mathrm{a}}$, alterations of global epigenetic and metabolic profiles, resistance to apoptosis, increases in autophagy, and implementation of a complex and multicomponent secretome known as the senescence-associated secretory phenotypes (SASP) (Campisi and Robert, 2014; Coppe et al., 2008; Munoz-Espin and Serrano, 2014; Rodier and Campisi, 2011). Through SASP, senescent cells produce a myriad of proinflammatory cytokines, chemokines, growth factors, and proteases (Kuilman and Peeper, 2009; Lasry and Ben-Neriah, 2015). These secreted molecules act through autocrine and paracrine signaling to not only reinforce the senescence state, but also non-autonomously affect 
neighboring cells in the tissue. However, it remains to be experimentally tested whether neurons, which are replication-incompetent by nature, can acquire the additional characteristics of senescent cells upon chronic DNA damage to promote neuroinflammation and neurodegeneration.

During studies of genome instability on cerebral cortical neurogenesis, we identified Brap as a key molecule to interact with essential genes for human brain development and brain genome protection (Houlihan and Feng, 2014; Lanctot et al., 2013). BRAP/Brap is a ubiquitously expressed cytoplasmic protein originally cloned through its interaction with the BRCA1 tumor suppressor (Li et al., 1998). It was also found to act as an ubiquitin E3 ligase and an effector of activated RAS (Matheny et al., 2004). Our previous study of central nervous system neurogenesis has shown a multifaceted role of Brap in modulating cell signaling downstream of RAS in a context-dependent fashion (Lanctot et al., 2013). Additionally, the E3 ligase activity of Brap allows it to serve as a liaison between the cell's environment and cell nucleus by targeting other E3 ligases with a wide-range of nuclear substrates (Lanctot et al., 2017). Moreover, the essential role of Brap goes beyond development. In a recent genome-wide association study of 500,000 genotyped individuals along with information on their parents' age and cause of death, the BRAP gene locus was linked to the human lifespan (Timmers et al., 2019). This has suggested a functional contribution of BRAP to human aging.

By analyzing Brap null $\left(\right.$ Brap $\left.^{-/}\right)$and cerebral cortical neural progenitor cell (NPC) Brap conditional knockout (Brap ${ }^{\text {cKONPC }}$ ) mice, we have shown that Brap loss of function (LOF) skews the cell cycle kinetics of multipotent NPCs, resulting in abrogation of the G1/S checkpoint and impeded generation of cerebral cortical neurons (Lanctot et al., 2017). We now demonstrate that besides the functional exhaustion of neural stem/progenitor cells, Brap ${ }^{\mathrm{cKONPC}}$ mice also present 
persistent DNA double-strand breaks (DSBs), increased cellular senescence, and significantly decreased life expectancy. We show that the accelerated aging of Brap ${ }^{\mathrm{cKONPC}}$ mice is caused by neuroinflammation and neurodegeneration along with remarkable cerebral cortical proteinopathy. We also show that DSBs in cortical neurons of Brap ${ }^{\mathrm{cKONPC}}$ mice elevate Brca1 as well as histone $\mathrm{H} 2 \mathrm{~A}$ ubiquitination (H2Aub) catalyzed by Brca1 E3 ubiquitin ligase. We further show that the high H2Aub can initiate histones proteolysis, leading to global chromatin changes, a backlog of ubiquitinated proteasome substrates, and senescence reinforcement. These data collectively suggest that histone $\mathrm{H} 2 \mathrm{Aub}$, as a mediator of genome instability and epigenetic alteration, is a key node connecting multiple aging hallmarks. As we also demonstrate that $\mathrm{H} 2 \mathrm{Aub}$ is elevated in cortical tissue from patients with Alzheimer's disease (AD), the results of this study highlight an essential impact of chromatin aberrations on aging and NDs.

\section{RESULTS}

\section{Brap LOF results in accelerated cellular senescence with persistent DSBs}

To further understand the mechanism by which Brap LOF results in skewed NPC cell cycle kinetics and differentiation that we observed previously, we isolated NPCs from Brap ${ }^{-/-}$embryos (before embryonic lethality by E13.5) and studied them in culture as neurospheres. Although $\mathrm{Brap}^{-/-}$NPCs grew at a rate comparable to their wild-type (WT) counterparts in the first 4-5 days in vitro (div), we failed to maintain them in culture as they abruptly ceased proliferation after 7-8 days (Figure 1A). This growth arrest was not accompanied by increased apoptosis (Figure 1B), which suggested that the mutant NPCs reached their replicative lifespan due to cellular senescence. 
To determine cellular senescence we turned to mouse embryonic fibroblast (MEFs) as they are better established for senescence analyses. We isolated MEFs from Brap ${ }^{-/}$and WT embryos and cultured them following the $3 \mathrm{~T} 3$ cell protocol (Todaro and Green, 1963). As expected, a greater fraction of Brap ${ }^{-/}$MEFs showed multiple essential characteristics of cellular senescence as early as passage 2 (P2). These include an enlarged cell body, high senescenceassociated beta-galactosidase (SA- $\beta$-gal) activity, and loss of the Ki67 proliferation marker, and the upregulation of $\mathrm{p} 19^{\text {Arf }}$, a CKI encoded by the $\mathrm{p} 16^{\mathrm{Ink4a}}$ locus but alternatively transcribed and can act together with $\mathrm{p} 16^{\text {Ink4a }}$ to induce cellular senescence (Capparelli et al., 2012; Haber, 1997; Quelle et al., 1995) (Figure 1C-F). We further validated the senescence phenotype of Brap LOF by examining the loss of Lamin B1, another characteristic of cellular senescence (Freund et al., 2010). Both immunofluorescence (IF) and immunoblotting (IB) analyses demonstrated that Lamin B1 was downregulated much more rapidly in Brap ${ }^{-/-}$than WT MEFs from P1 to P3 (Figure 1G, H). To acquire an ultimate proof of cellular senescence, we assessed several known SASP molecules, including Mmp1, Mmp2, Serpin1 (Pai1), and Osteopontin/Spp1 (OPN) (Aoshiba et al., 2013; Castello et al., 2017; Coppe et al., 2010; Flanagan et al., 2018; Ghosh and Capell, 2016; Pazolli et al., 2012; Rao and Jackson, 2016; Vaughan et al., 2017). We found that all of them were elevated in $\mathrm{Brap}^{-/-}$MEFs along with increased phosphorylation of NFkB p65 ${ }^{\mathrm{S} 536}$, a chief mediator for SASP(Salminen et al., 2012) (Figure 1I). The multiple essential features of cellular senescence shown by Brap ${ }^{-/}$cells demonstrates unambiguously that accelerated cellular senescence is the consequence of Brap LOF. These data also suggest that the reduced stemness of Brap ${ }^{-/}$NPCs was caused by a pro-senescence fate change.

While cellular senescence can be caused by a variety of factors, we found that it was associated with persistent DNA damage responses (DDRs) in Brap deficient cells and tissues. 
First, we detected significant elevations of phospho-p53, -Atm, -Atr, and 53BP1 in Brap ${ }^{-/-}$MEFs as early as in P1 (Figure 2A-C). In addition, a large fraction of Brap $^{-/-}$MEFs showed persistent DNA damage foci with high levels of $\gamma \mathrm{H} 2 \mathrm{~A} . \mathrm{X}$, the hallmark of DNA double-strand breaks (DSBs), and 53BP1, a marker for DSBs repair (Panier and Boulton, 2014; Thiriet and Hayes, 2005)(Figure 2D-F). Notably, the large $\gamma \mathrm{H} 2 \mathrm{~A} . \mathrm{X}$ and 53BP1 foci were in both proliferating (Ki67+) cells and cells that had ceased dividing (Ki67-) (Figure 2D and E, high magnification panels), indicating that Brap LOF can cause intractable DSBs that persist into cells that had undergone replication arrest. Furthermore, we found that the rapid progression to senescence of Brap $^{-/-}$MEFs coincided with increased level of $\gamma \mathrm{H} 2 \mathrm{~A} . \mathrm{X}$ ubiquitination (Figure $2 \mathrm{~F}$ ). As the ubiquitinated moiety of $\gamma \mathrm{H} 2 \mathrm{~A}$.X was shown to mark non-apoptotic DSBs and/or induced by oxidative DNA damage (Luczak and Zhitkovich, 2018), the ubiquityl- $\gamma \mathrm{H} 2 \mathrm{~A} . \mathrm{X}$ elevation and further enhancement with $\mathrm{H}_{2} \mathrm{O}_{2}$ treatment in $\mathrm{Brap}^{-/}$cells not only agrees with their evasion of apoptosis (Figure 2F), but also may mediate an unique senescence-prone DNA damage response of Brap LOF.

\section{Elevated cellular senescence and DSBs in cerebral cortical tissue underlie shortened lifespan of Brap ${ }^{\text {cKONPC }}$ mice}

We subsequently found that the accelerated cellular senescence and persistent DSBs shown by Brap $^{-/-}$cells in culture also occurred in vivo, leading to shortened lifespan of Brap mutant mice. We followed a cohort of adult Brap ${ }^{\text {flox Emx1Cre+ }}$ mutant mice (referred to as Brap $\left.{ }^{\text {cKONPC }}\right)(n=46)$ and their Brap ${ }^{\text {flox/WT }}$ or Brap ${ }^{\text {flox/flox }}$ Cre- control littermates $(n=29)$ over 12 months. We found that most Brap ${ }^{\text {cKONPC }}$ mice die between 4 and 8 months with a median lifespan of 6 months (Figure $2 \mathrm{G})$, whereas the average lifespan of mice is 2 years. The mutant mice lost weight, became 
lethargic, and showed slow or labored breathing when death became imminent (Supplementary Video). Since the expression of Cre recombinase in Brap ${ }^{\mathrm{cKONPC}}$ was restricted to NPCs of the dorsal telencephalon that give rise to glutamatergic neurons, astroglia, and oligodendrocytes of the cerebral cortex (Gorski et al., 2002), this result demonstrates that cerebral cortex deterioration can accelerate aging and shorten the lifespan.

We further showed that the midlife mortality of Brap ${ }^{\text {cKONPC }}$ mice was associated with cellular senescence in the cerebral cortical tissue. First, higher SA- $\beta$-gal activity was shown in many mutant cortical cells with the morphology of pyramidal neurons (Figure $2 \mathrm{H}$, arrows). This suggested that, despite being replication-incompetent, neurons can undergo additional senescence-associated chromatin and metabolic changes due to Brap LOF. Further corroborating with increased cellular senescence, the cortical tissue Brap ${ }^{\mathrm{cKONPC}}$ mice showed upregulated p16 $6^{\text {Ink4 }}$ expression, decreased level of Lamin B1, and increased SASP factors including Serpin1 (Pai-1), Mmp2, Ssp1 (OPN), and phospho-NFkB (Figure 2 I and J). Therefore, cellular senescence is also a phenotype of Brap LOF in the cerebral cortical tissue and the likely cause for the accelerated aging and premature death of the Brap ${ }^{\mathrm{cKONPC}}$ mice.

Similar to what we observed in Brap ${ }^{-/-}$MEFs, persistent DSBs with increased ubiquityl$\gamma \mathrm{H} 2 \mathrm{~A} . \mathrm{X}$ were distinctively shown in Brap ${ }^{\mathrm{cKONPC}}$ cortices. By IB analyses of histone extracts from the cortical tissues of adult Brap ${ }^{\mathrm{cKONPC}}$ and control mice, we found that $\gamma \mathrm{H} 2 \mathrm{~A} . \mathrm{X}$ in the Brap $^{\text {cKONPC }}$ cortices was not only elevated but also mono- and poly-ubiquitinated, showing a dominant pool of approximately $60 \mathrm{kDa}$ that is equivalent to the molecular weight of $\gamma \mathrm{H} 2 \mathrm{~A} . \mathrm{X}$ with Penta-ubiquitin conjugations (Figure $2 \mathrm{~K}$ ). Coincided with increased $\gamma \mathrm{H} 2 \mathrm{~A} . \mathrm{X}, 53 \mathrm{BP} 1$ was at higher levels in Brap ${ }^{\mathrm{cKONPC}}$ than in control cortical tissues at all postnatal ages, indicating that DSBs in the mutant cortex were persistent (Figure 2L). We further found $\gamma \mathrm{H} 2 \mathrm{~A} . \mathrm{X}$ 
immunoreactivities were restricted in the nucleus of a substantial fraction of cortical neurons $(\mathrm{NeuN}+)$ in the brain Brap ${ }^{\mathrm{cKONPC}}$ mice but was neither in mutant glia $(\mathrm{NeuN}-)$ nor in the brain of WT mice (Figure 2M). Given that neurons cannot replicate and we did not observe tumors or elevated apoptosis in the Brap ${ }^{\mathrm{cKONPC}}$ brain (Figure $2 \mathrm{~N}$ ), our findings further support the notion that senescence can be a major consequence of chronic DSBs in brain neurons and a negative determinant of the brainspan.

\section{Transcriptomic profiles of Brap ${ }^{\mathrm{cKONPC}}$ cortices reveal SASP candidates, immune activation, and impaired synaptic signaling}

Compared to apoptosis, senescence following unsuccessful DSB repair is more deleterious in vivo, as senescent cells are stably viable and can chronically influence neighboring cells by secreting soluble molecules or by altering cell surface and extracellular matrix (ECM) proteins. Although many senescence associated secretory molecules have been identified in various culture and in vivo models, the SASP profile of cellular senescence in the brain has not been investigated. We thus sought to assess senescence associated brain secretome by identifying molecules of which the expression was increased by Brap LOF in the cortical tissue. RNA sequencing (RNA-seq) was carried out with cerebral cortical total transcripts of Brap ${ }^{\text {cKONPC }}$ and littermate control mice of 3 months old. We identified 811 differentially expressed genes (DEGs) between the Brap ${ }^{\text {cKONPC }}$ and the control group, and 373 genes were significantly upregulated by Brap LOF (Figure 3A, Supplementary Table 1). Consistent with the pro-inflammatory feature of cellular senescence, we found 67 of the upregulated genes encode molecules associated with cells of the immune system and/or regulators of the innate immune response (Figure $3 \mathrm{~B}$ ). The upregulation of these genes indicates inflammation in the mutant brain tissue. Moreover, 80 
upregulated genes encode secreted molecules, which represent the cerebral cortical secretome of Brap ${ }^{\mathrm{cKONPC}}$ mice. Increased expression of these molecules can pose non-cell autonomous effects via SASP. These SASP candidate genes include not only immune active molecules and proteases but also diverse neuropeptide neurotransmitters and peptide hormones with potent activities in neural networking and cerebrovascular regulation (Figure 3C). An additional 83 upregulated genes encode plasma membrane and ECM proteins that interphase cells with their environment (Figure 3D). Increased expression of these genes can alter intercellular communication and tissue homeostasis. The most significantly upregulated genes in Brapc ${ }^{\text {KONPC }}$ cortices also include regulators for neurotransmitter metabolism, fatty acid and cholesterol metabolism, protein synthesis and sorting, as well as calcium homeostasis (Figure 3A, Supplementary Table 1). These molecular activities can have broad impacts on the brain tissue, resulting in a global alteration in brain immunity and neural function.

Brap LOF also resulted in decreased expression of 438 genes in the cortical tissue (Supplementary Table 1). Notably, the most significant downregulation occurred in genes that play key roles in regulating energy homeostasis, neuronal activity, synaptic plasticity, neuronal excitatory-inhibitory balance, Wnt signaling, retinoid metabolism, as well as the production, secretion, and activity control of neuropeptides or neurotransmitters (Figure 3A). We performed Gene Ontology (GO) enrichment analysis of the 50 most significantly downregulated genes in Brap $^{\text {cKONPC }}$ cortices. The GO terms identified in the BP category include impaired biological functions mainly in synaptic transmission as well as in cellular responses to stimuli and neuronal morphogenesis (Figure 3E). Overall, our unbiased transcriptomic data provide additional support for an impact of neuronal DSBs and cellular senescence on neuroinflammation and neural dysfunction of Brap ${ }^{\mathrm{cKONPC}}$ mice. 


\section{Neuroinflammation and neurodegeneration in Brap ${ }^{\mathrm{cKO}}$ brain}

Given that neuroinflammation is not only a common feature of senescence and tissue aging but also a key contributor to neurodegeneration, we asked whether DSBs and cellular senescence cause neurodegeneration. As expected, neuroinflammatory and neurodegenerative phenotypes were readily detectable in Brap ${ }^{\mathrm{cKONPC}}$ cortices starting at 3 months and progressing rapidly with age. First, hyperphosphorylation of the microtubule-associated protein tau became significantly increased in the cortical tissue of Brap ${ }^{\mathrm{cKONPC}}$ mice at 3 months and was further elevated as the mutant mice grew older (Figure 4A and B). Increased tau phosphorylation in Brap ${ }^{\text {cKONPC }}$ cortices was found on multiple residues including T181, T217, S396, and S416 (Figure 4A). The hyperphosphorylation on these residues is known to be associated with insoluble tau aggregates and neurodegenerative diseases. As the presence of these human tauopathy-like changes in Brap ${ }^{\text {cKONPC }}$ mice was at ages when mortality started to occur, it suggested that the mutant mice died of neurodegenerative brain dysfunction.

We further found astrogliosis and microgliosis were markedly elevated in Brap ${ }^{\text {cKONPC }}$ cortical tissue in an age-dependent fashion. Astrogliosis was undetectable in the cerebral cortex of Brap ${ }^{\text {cKONPC }}$ mice until about 3 months of age, and it then further progressed and became remarkably high as the mutant mice aged rapidly to death (Figure 4C and D; Figure S1A). Parallel to astrogliosis was the strong increase in microgliosis in Brap ${ }^{\mathrm{cKONPC}}$ cortices. As the brain's resident innate immune cells, microglia remain in a ramified resting state in healthy brains and are activated by injury or pathological processes. Analyses with pan-microglia marker Iba and activated phagocytic microglia marker CD68 both showed that microglia in Brap ${ }^{\mathrm{cKONPC}}$ cortices were de-ramified, amoeboid-like, and activated (Figure 4E). To confirm 
neuroinflammation, we examined the expression of several key inflammatory molecules implicated in human NDs (Dewachter et al., 2002; Frost et al., 2019; Newcombe et al., 2018; Sarlus and Heneka, 2017), including C1q, TNF $\alpha$, TGF $\beta 1$, and Trem2. All of them were upregulated significantly in the cortical tissue of 3-month Brap ${ }^{\text {CKONPC }}$ mice relative to that of the age-matched WT and/or control mice (Figure 4F). The strong astrocyte and microglial activation along with increased inflammatory cytokines in Brap ${ }^{\text {cKONPC }}$ cortical tissue were in line with RNA-seq data, and they together demonstrate the neuroinflammatory and neurodegenerative brain dysfunction of Brap ${ }^{\mathrm{cKONPC}}$ mice.

To ascertain Brap LOF leads to neurodegeneration, we examined the brain structure of Brap ${ }^{\text {cKONPC }}$ mice that survived to 6 months or older. We first performed a Golgi-Cox stain to reveal the morphology of cortical neurons. While neurons in Brap ${ }^{\mathrm{cKONPC}}$ brains were grossly normal in dendritic and axonal morphology, the density of their apical dendritic spines was significantly decreased in cortical layer II/III pyramidal neurons compared to those in wild type or control mice, whereas changes in dendritic spine density were insignificant in mutant mice of younger ages (Figure 4G). These results fully agreed with the impaired synaptic transmission and neuronetwork activities in Brap ${ }^{\mathrm{cKONPC}}$ mice revealed by RNA-seq, further supporting an agedependent decline in cortical neuronal functions.

To assess whether the decline of brain function in Brap ${ }^{\text {cKONPC }}$ mice was also due to an age-dependent neuronal loss, we examined H\&E stained brain sections of a set of Brap ${ }^{\text {cKONPC }}$ mice $(n=8)$ that survived to 6 months or older. We found all mutant brains showed spongiform encephalopathy in the cortical gray matter (Figure 4H). The spongiform changes were widespread, resulting in striking soma deformation and loss of cortical neurons (Figure $4 \mathrm{I}$, and J; Figure S1B). While spongiform encephalopathy is characteristic for prion diseases, neuronal 
vacuolization was not associated with the accumulation of the prion protein Prp and was also absent in younger Brap ${ }^{\mathrm{cKONPC}}$ mice or control littermates (Figure S1C), indicating it was a noninfectious neuropathological lesion. Many spongiform vacuoles in Brap ${ }^{\mathrm{cKONPC}}$ cortices were surrounded by reactive astrocytes and microglia (Figure $4 \mathrm{~K}$ ), suggesting that it was a part of neurodegenerative pathology associated with chronic neuroinflammation.

To investigate the causal relationship between cortical neuronal DSBs and neurodegeneration in Brap ${ }^{\text {cKONPC }}$ mice, we tested whether neuronal-specific Brap LOF could recapitulate the phenotype of Brap LOF in NPCs. We generated and analyzed a Brap neuronal conditional knockout line by abrogating Brap in neurons of the postnatal cortex and hippocampus with a Thy1-Cre driver (Dewachter et al., 2002)(Brap ${ }^{\text {flox Thy1Cre+; }}$ referred to as Brap $\left.{ }^{\mathrm{cKONeuron}}\right)$. About half of the Brap ${ }^{\mathrm{cKONeuron}}$ mice (12 out of 21$)$ were found dead by 6 months. Analyses of Brap ${ }^{\text {cKONeuron }}$ brains revealed phenotypes indistinguishable from those of the Brap $^{\text {cKONPC }}$ mice with respect to increased DSBs, $\gamma \mathrm{H} 2 \mathrm{~A} . \mathrm{X}$ ubiquitination, cellular senescence, tau hyperphosphorylation, astrogliosis, and microgliosis (Figure S1D-I). This indicated that the astroglia and microglia activation in Brap ${ }^{\mathrm{cKONPC}}$ mice occurred non-cell-autonomously due to defective cortical neurons. The phenocopy of Brap ${ }^{\text {cKONeuron }}$ and Brap ${ }^{\text {cKONPC }}$ mice strongly supports the notion that sustained DSBs and senescence of cortical neurons can be a main source of neuroinflammation to induce neurodegeneration.

\section{Cellular senescence is associated with histone $\mathrm{H2A}$ ubiquitination and histone proteolysis}

DSBs, if not properly repaired, can lead to several alternative consequences including oncogenesis, apoptosis, and cellular senescence. As brain tumors were not found in Brap ${ }^{\text {cKONPC }}$ mice, we sought to unravel the mechanism by which persistent DSBs selectively cause cellular 
senescence while rendering apoptosis resistance and tumor suppression. The distinctive features of cellular senescence are global metabolic and secretory changes mediated by epigenetic alteration. We thus asked whether the increased ubiquityl- $\gamma \mathrm{H} 2 \mathrm{~A} . \mathrm{X}$, as a unique DNA damage response of Brap LOF (Figure 2F and K), drives the epigenetic alteration for senescence reinforcement. Although mono-ubiquitination of histone $\mathrm{H} 2 \mathrm{~A}$ and $\mathrm{H} 2 \mathrm{~A}$ variants can act in a sitespecific manner to facilitate transcription silencing and DNA repair, respectively (Uckelmann and Sixma, 2017; Wang et al., 2004), Brap LOF increased both mono- and poly-ubiquityl$\gamma$ H2A.X, which suggested a possibility of ubiquitin-mediated histone degradation. Proteolytic depletion of histones was shown in oncogene-induced senescent human IMR-90 cells (Ivanov et al., 2013), where the autophagy-lysosome pathway (ALP)-dependent histone clearance was found necessary for stabilizing the senescence state. Similar to observations in senescent IMR-90 cells, we found all major histones were progressively depleted as MEFs underwent senescence, while Brap ${ }^{-/}$MEFs lost histones more rapidly than the WT MEFs (Figure 5A). This suggested that reducing the total histone content is a necessary epigenetic alteration for cellular senescence in both human and mouse cells.

We next found the histone loss coincided with the existence of cytoplasmic histone particles in Brap ${ }^{-/-}$MEFs. These cytoplasmic histone particles were rarely associated with DNA, but frequently co-occurred with the extrusion of Lamin B1 from the nucleus to the cytoplasm, while the nuclear envelope remained intact (Figure 5B, Figure S2A-C). Like histones, the cytoplasmic extrusion and ALP-dependent clearance of Lamin B1 was shown to mediate cellular senescence (Dou et al., 2015). Consistent with these previous studies, we found the autophagic flux in Brap ${ }^{-/-}$MEFs was higher than in WT MEFs, as indicated by increased autophagy markers LC3BII and p62/SQSTM1 (Figure 5C and D). Furthermore, cells presenting higher p62 and/or 
LC3B were precisely those that showed reduced nuclear histones and the existence of cytoplasmic histone particles. However, different from previous studies, we detected little colocalization of immunoreactivities of cytoplasmic histone particles with p62 and/or LC3B (Figure 5E-G), raising a possibility that histone clearance in Brap ${ }^{-/-}$MEFs was through the ubiquitin proteasome system (UPS) as suggested by the increased polyubiquityl- $\gamma \mathrm{H} 2 \mathrm{~A} . \mathrm{X}$, and that the elevation of autophagic flux was secondary to increased UPS burden.

To test UPS-dependent histone proteolysis, we examined the cytoplasmic histone-p62 or histone-LC3B co-localization by blocking proteasome function. We detected the co-localization of cytoplasmic histone particles with p62 or LC3B in Brap ${ }^{-/-}$MEFs by treating cells with the proteasome blocker MG132 but not by the lysosome inhibitor chloroquine (Figure 5H and I). This indicates that senescent Brap ${ }^{-/-}$MEFs relied mainly on the UPS for histone clearance. To verify the UPS-dependent histone proteolysis, we extracted histones from Brap ${ }^{-/}$and WT MEFs and analyzed their UPS- or ALP-dependent ubiquitination state. We found that Brap LOF resulted in a specific increase in both mono- and poly-ubiquitinated histone H2A (mono- or polyH2Aub). MG132 decreased mono-H2Aub but resulted in marked accumulation of poly-H2Aub, whereas chloroquine only slightly increased poly-ubiquitinated histones (Figure 5J). Therefore, UPS is primarily responsible for histone $\mathrm{H} 2 \mathrm{~A}$ proteolysis in $\mathrm{Brap}^{-/-}$cells, in which mono-H2Aub acts as a primer for poly-H2Aub and proteasomal degradation. We did not observe changes in ubiquitin-modification of H2B, H3, H4, and H1 (Figure S2D). Although ALP-dependent histone clearance remains possible, UPS-dependent histone H2A degradation is likely the initial driver for histone degradation in Brap $^{-/-}$MEFs.

We did not detect alteration of proteasome $20 \mathrm{~S}$ core catalytic proteins (Figure 5C and D). However, as histones are among the most abundant proteins in eukaryotic cells, their proteolysis 
via UPS is likely to over-occupy proteasome's capacity, resulting in UPS blockage and a global proteostasis impairment. Consistent with this, we observed a remarkable buildup of polyubiquitinated proteins in $\mathrm{Brap}^{-/-}$MEFs as they progressed to senescence (Figure 5K). Therefore, the depletion of histones and the accumulation of a large amount of ubiquitinated cellular proteins in Brap $^{-/-}$MEFs can together lead to the global epigenetic and metabolic alterations that are necessary for the stable expression of a multitude of phenotypes to reinforce the senescence state.

\section{The hallmark phenotype of Brap LOF is H2Aub mediated by BRCA1 activation}

Because we found H2Aub is pivotal for initiating histone proteolysis, we further evaluated whether it is a primary defect of Brap LOF by examining multiple tissues and ages of Brap mutant mice. We found H2Aub was significantly elevated in all cells, organs, and ages of Brap ${ }^{-/-}$ and Brap ${ }^{\mathrm{cKONPC}}$ mice relative to their wild type and control counterparts (Figure 6A; Figure $\mathrm{S} 3 \mathrm{~A})$. Therefore, increases in histone H2Aub is a hallmark phenotype of Brap LOF.

The ubiquitination of histone $\mathrm{H} 2 \mathrm{~A}$ is mediated by three E3 ligase complexes in a sitespecific manner. While the ubiquitin conjugation on K119 is the most prevalent form catalyzed by the polycomb repressive complex 1 (PRC1) (Tamburri et al., 2020), H2A can also be monoubiquitinated on K13 and K15 by RNF168/RNF8 or on K125, K127, and K129 by BRCA1 in response to DNA damage (Horn et al., 2019; Kalb et al., 2014; Uckelmann and Sixma, 2017). As histone H2AubK119 IBs only revealed a moderate increase in Brap deficient cells or tissues relative to controls (Figure 5J, Figure 6A, and Figure S3A), H2A in Brap deficient cells and tissues must be additionally ubiquitinated on residues other than K119 by RNF168 and/or BRCA1. 
Although BRAP resides in the cytoplasm, it can control nuclear proteins either by interacting with importin $\alpha$ and the nuclear localization signal (NLS) or by acting as an E3 ligase to regulate other E3 ligases with nuclear targets (Lanctot et al., 2017; Li et al., 1998). This makes it possible for Brap to regulate $\mathrm{H} 2 \mathrm{~A}$ ubiquitination by controlling the nuclear localization or the turnover of histone H2A E3 ligases. To test this, we first examined the level and nuclear localization of Brcal given its direct interaction with Brap. As expected, we found Brcal's abundance in Brap ${ }^{-/-}$MEFs, NPCs, embryos, as well as postnatal and adult Brap ${ }^{\text {cKONPC }}$ cortices was consistently increased in comparison to WT and control cells or cortices (Figure 6B). In addition, we found the nuclear pool of Brcal was much higher in Brap ${ }^{-/-}$than in WT cells (Figure 6C). The high level of nuclear Brcal was also shown by cortical neurons of the Brap ${ }^{\text {cKONPC }}$ mice (Figure 6D, Figure S3B). The increased nuclear Brcal abundance was not only in line with the original identification of BRAP as BRCA1's cytoplasmic docking protein but also suggested that Brcal was the DNA damage response-mediator in cells and tissues with Brap LOF. The E3 ligase activity of BRCA1 is integral for BRCA1's role in tumor suppression, though it appears to be independent of the well-recognized function of BRCA1 in DSB repair (Wu et al., 2009). Besides canonical H2A, the BRCA1 E3 ligase can target several H2A variants, including H2A.X and H2A.Y (MacroH2A1)(Kim et al., 2017). Since H2A.X and H2A.Y were also ubiquitinated at higher levels in Brap ${ }^{\mathrm{cKONPC}}$ than in control mice (Figure 6E, Figure S3C), these data strongly suggest that increased Brca1 E3 ligase activity underlies the elevated H2Aub resulting from Brap LOF.

Compared to the robust change in Brcal, alterations in the level and/or nuclear localization of PRC1 and RNF168 by Brap LOF were subtle or undetectable. We also found RNF168 as well as the PRC1 components Ring1B and Bmi-1 were much more abundant in 
developing than in aging brains. While a slightly higher level of Ring1B and Bmi-1 was observed in line with the moderate increase in H2AubK119 in Brap ${ }^{\text {cKONPC }}$ than in control cortices, we failed to detect an upregulation of RNF168 in Brap mutants (Figure S3D and E). Collectively, these results suggest that Brca1 is mainly responsible for $\mathrm{H} 2 \mathrm{Aub}$ resulting from Brap LOF. This BRCA1 E3 ligase activity may contribute to tumor suppression by driving cells with persistent DSBs to senescence.

\section{H2Aub is coupled with loss of proteostasis in cerebral cortical tissue and is elevated in}

\section{Alzheimer's disease}

To determine whether increased histone $\mathrm{H} 2 \mathrm{Aub}$ can drive brain aging and promote neurodegeneration, we further examined the histone content, ubiquitinated protein levels, and protein homeostasis in cortical tissues of Brap ${ }^{\text {cKONPC }}$ mice. Reminiscent of senescent Brap ${ }^{-/-}$ MEFs, increased mono-H2Aub in Brap ${ }^{\text {cKONPC }}$ cortices was accompanied by poly-H2Aub buildup and nuclear histone reductions in a subpopulation of cells (Figure 6F and G). We found cells showing weaker nuclear histone immuno-reactivity were surrounded by reactive astrocytes, supporting the notion that histone proteolysis stabilizes the senescent cell state and mediates the senescence-associated neuroinflammation. Also similar to what was observed in Brap ${ }^{-/-}$MEFs, increased mono- and poly-H2Aub was accompanied by substantial elevation of the overall level of poly-ubiquitinated proteins in the Brap ${ }^{\text {cKONPC }}$ cortical tissue. While the overall level of polyubiquitinated proteins in Brap ${ }^{\mathrm{cKONPC}}$ cortices were not obviously altered during embryonic and neonatal development, it started to elevate at young adult ages, then further built up progressively with age, and became remarkably high in all Brap ${ }^{\mathrm{cKONPC}}$ mice by 6 months of age (Figure 7A and B; Figure S4A and B). The accumulation of a large amount of poly-ubiquitinated 
proteins, in conjunction with the high level of H2Aub, indicates compromised UPS function.

This results in widespread proteopathy in Brap ${ }^{\text {cKONPC }}$ cortices, impairing brain functions at multiple levels.

Because the progressive decline in proteostasis is a prominent hallmark of aging and a common pathological feature of neurodegeneration, we assessed the altered cortical proteome in aged cortical tissue of Brap ${ }^{\mathrm{cKONPC}}$ mice to evaluate whether it may resemble the proteopathy of NDs. We performed tandem mass tags (TMT) quantitative proteomics analysis of whole cortical proteins from 6-month old Brap ${ }^{\mathrm{cKONPC}}$ and control mice. Out of the total 4708 quantified proteins, the abundance of 903 proteins was significantly altered by Brap LOF (Supplementary Table 2). Notably, many toxic proteins associated with Alzheimer's disease (AD), Parkinson's disease (PD), Huntington's disease (HD), and Amyotrophic lateral sclerosis (ALS) were found accumulated in the Brap ${ }^{\mathrm{cKONPC}}$ relative to the control cortical tissues (Figure 7C), which suggested overlapping pathogenesis between Brap LOF and human NDs. We further analyzed the alteration in proteomic profiles resulting from Brap LOF by testing their association with biological processes. In addition to aberrant protein ubiquitination and quality control, Ingenuity Pathway Analysis (IPA) showed that the cerebral cortex of Brap ${ }^{\mathrm{cKONPC}}$ mice had altered glucose and fatty acid metabolism, deregulated nutrient sensing, as well as declines in numerous cell signaling pathways and neuronal network activities (Figure 7D). Notably, both long-term depression (LTD) and long-term potentiation (LTP) were significantly decreased in Brap ${ }^{\text {cKONPC }}$ cortices, indicating diminished synaptic plasticity and impaired cognition of the mutant brain (Figure 7D). These data provided strong evidence for the brain functional decline of Brap ${ }^{\text {cKONPC }}$ mice before death. While the level of most proteasome 20S catalytic core proteins remains unaltered, we found multiple components of the $19 \mathrm{~S}$ proteasome regulatory particles were 
significantly upregulated in Brap ${ }^{\text {cKONPC }}$ relative to control cortices (Figure S4C), which supported the increased UPS burden in the mutant. Besides increased ubiquitination of canonical H2A, the abundance of multiple histone H2A variants, including H2A.X, H2A.Y, H2A.Z, H2A.W, and H2A.2B, was also significantly higher in Brap ${ }^{\mathrm{cKONPC}}$ than in control cortices (Figure 7E). The significant changes in $\mathrm{H} 2 \mathrm{Aub}$ and multiple histone $\mathrm{H} 2 \mathrm{~A}$ variants reflect the substantial chromatin remodeling in the Brap ${ }^{\text {cKONPC }}$ brain. They support the notion that aging and neurodegeneration can be initiated by epigenetic alterations of histone $\mathrm{H} 2 \mathrm{~A}$ and $\mathrm{H} 2 \mathrm{~A}$ variants.

The overlapping transcriptomic and proteomic alterations between Brap ${ }^{\text {cKONPC }}$ mice and NDs prompted us to determine whether the primary defect caused by Brap LOF may underlie human brain aging and neurodegeneration, as there is an urgent need for animal models to uncover the origin and recapitulate the progression of NDs. To test whether our findings were applicable to human conditions, we acquired postmortem brain samples of three Alzheimer's disease $(\mathrm{AD})$ patients along with age-matched normal controls to study their histone levels and histone ubiquitination states (Supplementary Table 3). Intriguingly, our analysis of histone extracts from the human tissue specimen demonstrated that, compared to histone $\mathrm{H} 2 \mathrm{~A}, \mathrm{H} 2 \mathrm{~B}, \mathrm{H} 3$, or $\mathrm{H} 4$, the level of histone $\mathrm{H} 2 \mathrm{Aub}$ was overall higher in the cortical tissue of all three AD than in that of control individuals (Figure 7F; Figure S4D, E). The abundance of mono- and/or poly$\mathrm{H} 2 \mathrm{Aub}$, albeit variable in different $\mathrm{AD}$ individuals and detection conditions, was notably increased in $\mathrm{AD}$ relative to control brains. All three $\mathrm{AD}$ specimens presented a marked enrichment of the $60 \mathrm{kDa}$ Penta-ubiquitinated H2A (H2Aub5), while increases in H2AubK119 in AD brains were relatively subtle. Moreover, similar to our observations of Brap ${ }^{\mathrm{cKO}}$ mice, polyubiquitinated histones were found at a higher level in the cortical tissue of AD than in normal age-matched individuals (Figure 7F). The close resemblance in histone ubiquitination between 
$\mathrm{AD}$ and $\mathrm{Brap}^{\mathrm{cKO}}$ mice supports the relevance of our data to human NDs. It also suggests that $\mathrm{H} 2 \mathrm{Aub}$ is a common epigenetic modification associated with brain aging and neurodegeneration, and that the Brap ${ }^{\mathrm{cKO}}$ mouse model can be useful for studying AD and NDs.

\section{DISCUSSION}

In this study, we show that Brap LOF causes accelerated aging at the cellular, tissue, and organismal levels, resulting in significant lifespan shortening in mice. This finding is consistent with the results of recent large-scale GWAS that identified the association of BRAP locus with human lifespan. They together suggest that a better understanding of BRAP's gain and loss of function would provide insight into the molecular determinants for aging and longevity. Moreover, our studies of the cerebral cortical NPC- and neuron-specific Brap conditional knockout mice reveal a histone H2Aub-based mechanism that connects multiple aging hallmarks to neurodegeneration, serving as a new model for aging-associated diseases.

\section{The interconnection of multiple aging hallmarks underpins the complex etiology of aging-}

\section{associated diseases}

Aging is an inevitable natural process driven by numerous molecular and cellular events.

However, each cellular or molecular event, per se, is insufficient to cause morbidity and mortality. At the systemic level, multiple aging promoting mechanisms must act synergistically to cause irreversible functional decline of organs involved. Our findings suggest that BRAP is a multifunctional molecule capable of connecting incoming signals that cells receive to cells' functional outputs governed by chromatin. The E3 ligase activity of BRAP makes it a versatile, yet powerful, molecule that is capable of controlling the levels of many cellular proteins through 
modifying the ubiquitination of itself and other E3 ligases with broad substrates. Therefore, it is not surprising that Brap LOF affects a myriad of cellular proteins and pathways, presenting a multitude of hallmark features of aging including stem cell exhaustion, genome instability, epigenetic alteration, cellular senescence, loss of proteostasis, as well as deregulated nutrient sensing, cell signaling, and cell communication.

Through the model of Brap LOF we have learned that the abnormal increase in histone $\mathrm{H} 2 \mathrm{Aub}$ can serve as a nexus for multiple aging accelerating mechanisms. Histone H2A monoubiquitination has been implicated as an evolutionarily conserved aging biomarker from an ubiquitylome study of long-lived Drosophila proteins. It was shown to increase in an agedependent manner not only in Drosophila but also in the brain of mice, non-human primates, and humans (Yang et al., 2019). However, the study attributed such an increase solely to H2AubK119 based on its long life in Drosophila, whereas our results partially echo this result by showing a moderate increase of H2AubK119 in Brap mutant mice. Our additional findings demonstrated that the gained $\mathrm{H} 2 \mathrm{Aub}$ in the aging Brap $^{\mathrm{cKO}}$ mouse and $\mathrm{AD}$ brains was also outside of K119. Moreover, instead of maintaining H2A stability, we show that mono-H2Aub can lead to poly-H2Aub and UPS-mediated histone proteolytic degradation. This ubiquitin-dependent histone degradation not only results in global epigenetic alteration but also increases the UPS burden, leading to proteopathy and an irreversible senescent state in cells being affected.

It is especially intriguing that the overall ubiquitination level of $\mathrm{H} 2 \mathrm{~A}$ is increased in the cortical tissue of AD. Although the terminal brain pathology of AD is characterized by the accumulation of amyloid- $\beta$ and hyperphosphorylated tau proteins, the cause of idiopathic AD remains elusive, though aging is known as its major risk factor. Our finding here suggests that histone $\mathrm{H} 2 \mathrm{Aub}$ is a potential biomarker for $\mathrm{AD}$, since the level of $\mathrm{H} 2 \mathrm{Aub}$ may mark cells' or 
organs' pathophysiological age instead of chronological age by connecting chromatin aberration to cellular senescence and proteinopathy. While H2Aub-mediated epigenetic and proteomic changes cause profound cell-intrinsic phenotypes, the senescence-associated autocrine and paracrine signaling can result in tissue-wide changes and immune-activation, leading to brain deterioration at multiple levels. Therefore, epigenetic aging associated with H2Aub may underlie NDs and other aging-associated diseases.

\section{The senescent states are diverse, context-dependent, and can apply to neurons}

Cellular senescence, defined traditionally as a cell that can no longer divide, is essentially a stress response to reactive oxygen species, DNA damage, and protein misfolding or aggregation. However, cellular senescence presents many acquired characteristics beyond cell cycle arrests, such as metabolic activation and SASP. Although NPCs and MEFs with Brap deficiency undergo canonical cellular senescence in culture, the senescent phenotype in cortical tissues of Brap $^{\mathrm{cKO}}$ mice is more complex. Despite increases in bona fide senescence markers of SA- $\beta$-Gal and $\mathrm{p} 16^{\text {Ink4a }}$, there was no replication arrest of any type of brain-resident cells in the Brap ${ }^{\mathrm{cKONPC}}$ brain. Because the age-dependent neuroinflammation, proteopathy, brain structural deterioration, and mid-life mortality were linked to Brap LOF in neurons showing DSBs, it suggests that cellular senescence is dictated by the chromatin state. Such chromatin state not only entails the permanent cessation of propagating cells with irreparable damage but also elicits a multitude of damage responses, leading to the widespread transcriptome, metabolome, and proteome alterations for cells to adjust their microenvironment and their interaction with neighboring cells. Therefore, cellular senescence might be better defined by the distinctive chromatin state, metabolic pattern, and SASP. By this definition, neurons, which are intrinsically postmitotic, can 
further progress to a senescent state if they harbor sustained DSBs, leading to brain-wide metabolic and inflammatory changes.

Our RNA-seq with bulk cortical tissue identified 373 upregulated genes in Brap ${ }^{\text {cKONPC }}$ mice, of which 80 encode secreted proteins. While some of these transcripts could be from activated astrocytes, microglia, and lymphocytes, many are expected to represent primary SASP of Brap deficient cortical neurons with newly adopted epigenetic states. As the molecular responses of cellular senescence are context-dependent, the molecular composition of SASP is expected to be heterogeneous and pleiotropic, which can be both beneficial and destructive depending on the stage of phenotype progression. Further studies to identify primary SASP molecules from Brap deficient cortical neurons in young and aged mice, respectively, will provide in-depth information on the initiation and progression of neuroinflammation and degeneration.

\section{BRCA1-mediated histone H2Aub commits cells to cellular senescence}

Brap was originally cloned as a regulator of the BRCA1 tumor suppressor. It binds to the NLS of BRCA1 to control BRCA1's shuttling between the nucleus and the cytoplasm. Variations in BRCA1's nuclear vs. cytoplasmic localization have a strong correlation with aggressive tumor features and/or poor prognosis (Chen et al., 1995; Scully et al., 1996; Wilson et al., 1999). Nonetheless, the role of BRCA1 in tumor suppression is so far attributed only to the nuclear pool. The function of cytoplasmic BRCA1 remains unknown. This study showed that the cytoplasmic pool of Brca1 was decreased upon Brap LOF. The loss of cytoplasmic Brca1 coincides with higher nuclear and overall Brcal abundance, excessive histone H2Aub, cellular senescence, and accelerated aging. This implicates an anti-senescence and anti-aging role of the 
cytoplasmic BRCA1. Such function may be especially essential in stem cells and healthy somatic cells, whereas the nuclear BRCA1 pool dominates in cells with genomic lesions. This would well explain the requirement of Brca1 in development as well as the cellular senescence phenotype upon Brcal abrogation (Cao et al., 2003; Pulvers and Huttner, 2009). Further learning the conditions by which BRAP controls BRCA1's nuclear and cytoplasmic homeostasis is necessary to delineate the diverse roles of BRCA1 in development, aging, and oncogenesis.

Besides a major function in DSB repair, BRCA1 is well recognized by its ubiquitin E3 ligase function. Mutations within the N-terminal RING domain of BRCA1, which abrogates the E3 ligase activity, have been linked to breast and ovarian cancers. The BRCA1 E3 ligase activity was further found independent of its role in DSB repair but essential for tumor suppression (Reid et al., 2008; Wu et al., 2008; Zhu et al., 2011). Data of this study suggest that, by targeting histone $\mathrm{H} 2 \mathrm{~A}$ for ubiquitination and proteolysis, the BRCA1 E3 ligase may direct cells with chronic DSBs to senescence. Such action can stop the propagation of cells with irreparable DNA damage and prevent their oncogenic transformation. This mechanism can contribute significantly to BRCA1's role in tumor suppression, though it may be at the expense of exhausting stem cells and speeding up the aging of those cells or organs with increased genome instability.

The BRCA1 E3 ligase modifies the C-terminus of histone $\mathrm{H} 2 \mathrm{~A}$ and accounts for the gained H2Aub in Brap mutant cells. Although we do not rule out the possible contribution of other E3 ligases that may function redundantly with Brcal as the H2Aub writer, regulatory mechanisms involving H2A's C-terminus are of special interest. The histone H2A family contains numerous isoforms and variants, among which the highest degree of diversification is in their C-termini. H2A sits on the edges of the histone octamer with the flexible C-terminus tail protruding from the globular nucleosome particle at the DNA entry/exit site. The structure and 
variation of the $\mathrm{H} 2 \mathrm{~A} \mathrm{C}$-termini thus have strong influences on nucleosome stability and chromatin structure. The H2Aub produced by BRCA1 was implicated in heterochromatin compaction (Zhu et al., 2011), and chromatin condensation is also the result of histone degradation and replacement by transition proteins in spermatogenesis (Govin et al., 2004). At present, we do not know whether senescence or aging-associated histone proteolysis results in nucleosome-free DNA or histone replacements. However, it is intriguing that many histone H2A variants were increased in the "aged" Brap ${ }^{\text {cKONPC }}$ cortical tissue. This could be due to either compensation for the loss of canonical H2A or an overall change in the turnover of $\mathrm{H} 2 \mathrm{~A}$ variants. The end result of $\mathrm{H} 2 \mathrm{Aub}$ and $\mathrm{H} 2 \mathrm{~A}$ variants swap could underlie the chromatin response necessary for directing cells with persistent DSBs to senescence as opposed to oncogenesis or apoptosis. Additional insights on the altered nucleosome structure as well as the genomic loci involved in these chromatin responses will allow a better understanding of the cell fate regulation in response to genomic instability in the brain.

\section{Intrinsic sources of genome instability in cerebral cortical neurons}

DNA damage, particularly DSBs, have been shown to increase in brains of AD, PD, and ALS patients (Kim et al., 2020; Mitra et al., 2019; Schaser et al., 2019; Shanbhag et al., 2019). While genomic instability is considered a contributor in the pathogenesis of neurodegenerative disorders, the causes of neuronal DSBs and the mechanism by which DSBs induce the degenerative brain pathology remain to be defined. In the cerebral cortical tissue, Brap LOF resulted in chronic DSBs specifically in neurons. While this may be due to neurons' high metabolic rate, potent generation of reactive oxygen species, and abundant polyunsaturated fatty acids prone to peroxidation, we also believe neuronal DSBs are the consequence of aberrant 
chromatin remodeling in differentiation. Although Brap is expressed ubiquitously, Brap LOF specifically impairs the neuronal fate-restrictive cell cycles of NPCs during cortical development. Such defect in neurogenesis is likely to result in poor genome quality control of cortical neurons. Since neurons lack the ability of regeneration, the aberrant chromatin configuration acquired in neuronal differentiation is likely the main source for vulnerability to genotoxic insults throughout neurons' lifespan. We have observed remarkable nuclear architecture aberrations in Brap mutant NPCs and young neurons. These nuclear architecture aberrations mainly involve the constitutive heterochromatin composed of repetitive satellite DNA sequences. It is conceivable that unprotected satellite repeats can increase undesired recombination and DSBs that overwhelm the DNA damage repair machinery. Notably, chromatin immunoprecipitation (ChIP) analyses have shown that mono-H2Aub is enriched in the satellite regions of the genome (Padeken et al., 2019; Zhu et al., 2011). Therefore, the persistently high level of histone $\mathrm{H} 2 \mathrm{Aub}$ seen in Brap ${ }^{\mathrm{cKO}}$ mice may not only mark the damaged loci but also induce chromatin condensation. Consequently, the broad change in chromatin state can alter gene expression and metabolism to drive neurodegeneration.

\section{The deep chromatin root of neurodegenerative disorders and Alzheimer's disease}

Various NDs share common defects in the accumulation and aggregation of misfolded proteins despite the fact that each of them presents unique pathological hallmarks, specific brain regional involvement, and distinctive clinical symptoms. Tremendous effort has been made to target protein aggregates, especially the amyloid plaques in $\mathrm{AD}$, but the efficacy of these treatment regimens has been uncertain, suggesting that the aggregated proteins are the terminally pathological manifestations rather than the original cause of neurodegeneration. It is currently 
believed that $\mathrm{AD}$, and many other NDs, may have a long asymptomatic phase that lasts as long as decades, during which the diseases are frequently associated with chronic neuroinflammation and brain vascular dysfunction. The complex etiology of NDs has also made it difficult to establish proper animal models. None of the existing models of AD, PD, or ALS fully phenocopies the human disease conditions and progression. It is thus extremely challenging to identify the early diagnostic markers as well as the initial cellular and molecular defect that primes the progressive brain tissue-wide deterioration.

We believe the Brap ${ }^{\mathrm{cKO}}$ mouse model will be invaluable for understanding the molecular mechanism and early-phase pathogenesis of NDs and AD. The wide range of phenotypes presented by the Brap ${ }^{\mathrm{cKO}}$ mice is well in line with the disease states of human NDs. Its molecular profiles of neuroinflammation as well as gene expression and proteomic aberrations overlap substantially with hallmark pathologies of AD, PD, HD, and ALS. Therefore, data obtained from Brap $^{\mathrm{cKO}}$ mice are justified to serve as entry points for exploring the deep roots and complex etiology of neurodegeneration. As suggested, there is a chromatin origin of AD and NDs. Given that dynamic chromatin remodeling is an intrinsic need for neuronal plasticity, genome instability and epigenome aberrations are expected to have a constant and lifelong impact on neuronal function and longevity. A better understanding of chromatin quality control holds promises for the identification of early and effective targets for preventing and treating NDs.

\section{STAR METHODS}

\section{KEY RESOURCE TABLES}

Supplemental Table 1: RNA-seq Data Table

Supplemental Table 2: TMT Proteomics Data Table 
Supplemental Table 3: Human Postmortem AD and Control Brain Tissue Specimen Table Antibodies Table

Supplemental video

\section{EXPERIMENTAL MODEL AND SUBJECT DETAILS}

\section{Mice:}

Brap knockout $\left(\right.$ Brap $\left.^{-/}\right)$, floxed $\left(B_{\text {rap }}^{\text {flox/flox }}\right)$, and Emx1-cre mediated conditional knockout $\left(\right.$ Brap $\left.^{c K O N P C}\right)$ mice have been described previously (Lanctot et al., 2017). The Thy1-cre mice were purchased from JaxMice (Stock No: 006143) and used to generate Brap ${ }^{\text {cKONeuron by }}$ crossing with Brap ${ }^{\text {flox/flox }}$ mice. All mice used for this study were housed and bred according to the guidelines approved by the IACUC committees of Northwestern University and Uniformed Services University of Health Services in compliance with the AAALAC's guidelines.

Experiments were performed using littermates or age and genetic background matched control and mutant groups in both sexes. For timed matings, the day of vaginal plug was considered E0.5.

\section{Cell Culture}

Neural stem/progenitor cells were isolated from embryonic cortices at E12.5. Single cells were prepared and cultured in DMEM/F12 with N2 and B27 supplements, Penicillin-Streptomycin, Glutamine, Heparin, and growth factors (20 ng/ml EGF and $10 \mathrm{ng} / \mathrm{mL}$ FGF). Mouse embryonic fibroblasts (MEFs) were isolated from embryos at E12.5, after removing head and liver. The embryos were minced and digested in $0.25 \%$ trypsin-EDTA at $37^{\circ} \mathrm{C}$ with agitation for $10 \mathrm{~min}$. Single cell suspensions were plated at a density of $\geq 10^{4} / \mathrm{cm}^{2}$, which is considered passage 0 . 
The cells were then cultured according to the standard 3T3 cell protocol in DMEM supplemented

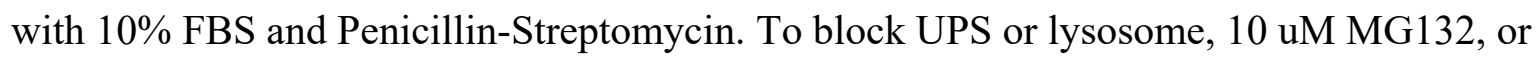
$25 \mathrm{mM}$ chloroquine were applied to MEF culture for 4-12 hours.

\section{METHOD DETAILS}

\section{Immunoblotting}

Immunoblotting of total cell or tissue proteins was performed by extracting with boiling 2x SDS PAGE sample buffer (62.5 mM Tris-HCl, pH 6.8, 2.5\% SDS, 0.7135 M $\beta$-mercaptoethanol, 10\% glycerol, $0.002 \%$ Bromophenol Blue) to fully dissolve the tissue proteins, heating at $95^{\circ} \mathrm{C}$ for $10 \mathrm{~min}$ to ensure protein denaturation, and passing through syringes with a $29^{1 / 2}$ gauge needle three times to sheer nuclear DNA and obtain homogenous extracts. 10-30 ug of total proteins were used for each immunoblotting analysis. Loadings were adjusted and normalized by the total protein content according to Coomassie blue stain of the gel after SDS PAGE and by the level of housekeeping proteins.

\section{Immunostaining, Immunofluorescence, and Immunohistological Analyses}

Immunofluorescence staining of cells and brain tissue sections was carried out as described (Lanctot et al., 2017; Lanctot et al., 2013). Briefly, cells were fixed with either 4\% formaldehyde or cold methanol, blocked in $1 \%$ BSA and $5 \mathrm{mg} / \mathrm{ml}$ lysine, and immuno-stained in a buffer containing 25 mM Hepes, pH 7.4, 250 mM Sucrose, 25 mM KCl, 25 mM Mg(CH 3 COO) $2,1 \%$ BSA, and $0.25 \%$ Saponin. Mouse brains were fixed by transcardial perfusion with PBS and $4 \%$ paraformaldehyde and then processed in 12 um cryosections or 5 um paraffin sections. After treating with antigen unmasking solutions (Vector Labs), brain sections were blocked with 5\% 
goat serum and incubated with primary antibodies in PBS, $0.05 \%$ Triton X100, and 5\% goat serum at $4{ }^{\circ} \mathrm{C}$ overnight, and followed by staining with fluorescence conjugated antibodies and Hoechst 33342. Epifluorescence images were acquired with a Leica CTR 5500 fluorescence, DIC, and phase contrast microscope equipped with the Q Imaging Regita 2000R digital camera. Images were imported to Adobe Photoshop and adjusted for brightness and black values.

\section{Senescence-associated $\beta$-gal (SA- $\beta$-gal) Staining}

For identification of cellular senescence, cells were fixed with $2 \%$ formaldehyde and $0.2 \%$ glutaraldehyde for $5 \mathrm{~min}$ at room temperature. Mouse brains were transcardially perfused and fixed with ice cold PBS followed by $4 \%$ paraformaldehyde and $5 \mathrm{mM} \mathrm{MgCl}_{2}$ in PBS for $6-8$ hours. The fixed brains were cryopreserved and for prepared as cryosections 16 um in thickness. Fixed cells or brain sections were stained with a staining solution containing $5 \mathrm{mM}$ Potassium Ferrocyanide, $5 \mathrm{mM}$ Potassium Ferricyanide, $150 \mathrm{mM} \mathrm{NaCl}, 5 \mathrm{mM} \mathrm{MgCl} 2,40 \mathrm{mM}$ Citric acidNa phosphate buffer $\mathrm{pH} 5.95$, and $1 \mathrm{mg} / \mathrm{ml} \mathrm{X-gal} \mathrm{at} 37^{\circ} \mathrm{C}$ for $6-20$ hours.

\section{Golgi-Cox Staining and Dendritic Spine Analysis}

Mice were anesthetized with $\mathrm{CO}_{2}$; brains were quickly dissected, rinsed with deionized water, immersed in impregnation solution, and processed using FD Rapid GolgiStain kit (FD NeuroTechnologies) according to manufacturer's instructions. Stained sections were examined under a Leica DM5000 light microscope. Pyramidal neurons in the cerebral cortex and hippocampus regions were imaged with 40x objective and photographed. For dendritic spine density analysis, 16-20 pyramidal neurons in neocortical layer II/III of each mouse were randomly selected for assessment. The number of spines per 10 micrometers in secondary apical 
dendrites (branched from primary dendrites arising from the soma) was scored using the NIH Image J software.

\section{Histone Extractions}

The brain tissues used in this project were provided by the University of California Irvine Alzheimer's Disease Research Center (UCI ADRC) and the Institute for Memory Impairments and Neurological Disorders (UCI MIND). Cell or tissues were re-suspended or homogenized and lysed in PBS containing 0.5\% Triton X 100, $25 \mathrm{ug} / \mathrm{ml}$ leupeptin, $10 \mathrm{ug} / \mathrm{ml}$ Pepstatin A, $5 \mathrm{ug} / \mathrm{ml}$ Aprotinin, $10 \mathrm{mM}$ Benzamidine, $2 \mathrm{mM}$ PMSF, 10mM N-Ethylmaleimide (NEM), 10mM iodoacetamide (IAA), and $0.02 \% \mathrm{NaN}_{3}$ at a density of $\sim 10^{7}$ cells $/ \mathrm{ml}$. Nuclei were first collected by centrifuge at $2500 \mathrm{x}$ f for $10 \mathrm{~min}$ at $4^{\circ} \mathrm{C}$, washed once, and re-suspend in $0.2 \mathrm{~N} \mathrm{HCl}$ at a density of $4 \times 10^{7}$ nuclei per $\mathrm{ml}$ to extract histones overnight at $4^{\circ} \mathrm{C}$. After clearing debris by centrifugation at $10,000 \mathrm{xg}$ for $10 \mathrm{~min}$ at $4^{\circ} \mathrm{C}$, the histone containing supernatants were neutralized with 2M Tris Base. Protein concentration was determined by measuring absorbance at $280 \mathrm{~nm}$. Histone extractions were stored in aliquots at $-20^{\circ} \mathrm{C}$.

\section{Cytoplasmic-nuclear Fractionation}

Cells grown on culture dishes were trypsinized, collected in centrifuge tubes with DMEM and $10 \% \mathrm{FBS}$, and washed with PBS. After complete removal of PBS, cells were first treated gently with hypotonic buffer (10 mM Hepes, $\mathrm{pH} 7.9,1.5 \mathrm{mM} \mathrm{MgCl}_{2}$, and $\left.10 \mathrm{mM} \mathrm{KCl}\right)$ and protease inhibitors on ice for $10 \mathrm{~min}$ before the addition of NP-40 to a final concentration of $0.1 \%$. After gently mixing, cells were spun at 2,500 x g for $10 \mathrm{~min}$ at $4{ }^{\circ} \mathrm{C}$. Supernatants were collected and analyzed as cytoplasmic fractions either directly or precipitated by $10 \%$ TCA. Pellets were 
gently washed twice with the hypotonic buffer by spinning at $2500 \mathrm{x} g$ for $5 \mathrm{~min}$ before being analyzed as nuclear fractions.

\section{RNA Isolation and Quantitative RT-PCR}

Cerebral cortical tissue was homogenized in TRIzol reagent (Thermo Fisher) followed by total RNA extraction according to the manufacturer's protocol. 1ug RNA was reverse transcribed into first-strand cDNA using Superscript III reverse transcriptase (Invitrogen). qRT-PCR reactions were performed using Power SYBR Green PCR Master Mix on Roche LightCycler 480 RealTime PCR system. Primers used for accessing gene expression were synthesized according to validated primer sequences from the MGH-PGA PrimerBank: p16Ink4a ( forward:

CGCAGGTTCTTGGTCACTGT; reverse: TGTTCACGAAAGCCAGAGCG), Pai-1 (forward: 5'-TTCAGCCCTTGCTTGCCTC; reverse: ACACTTTTACTCCGAAGTCGGT), TGF $\beta 1$ (ATGTCACGGTTAGGGGCTC; reverse: GGCTTGCATACTGTGCTGTATAG), Tnf( forward: CTGGATGTCAATCAACAATGGGA; reverse: ACTAGGGTGTGAGTGTTTTCTGT), C1qc ( forward: 5'-CCCAGTTGCCAGCCTCAAT-3'; reverse: GGAGTCCATCATGCCCGTC), Trem2 (forward: 5'CTGGAACCGTCACCATCACTC; reverse: CGAAACTCGATGACTCCTCGG), and Tbp ( forward: 5'- AGAACAATCCAGACTAGCAGCA; reverse:

GGGAACTTCACATCACAGCTC). Expression was normalized to TATA-binding protein (Tbp) as an internal control and results were analyzed using the $2^{-(\Delta \Delta \mathrm{CT})}$ method.

\section{RNA Sequencing Analysis of Whole Cerebral Cortical Transcriptome}


Purified total RNA from whole cerebral cortical tissue of five Brap ${ }^{\mathrm{cKONPC}}$ and five control (Brap $^{\text {flox/floxCre- }}$ and Brap ${ }^{\text {flox/WTCre- }}$ ) mice at 3 months of age were processed at the University of Chicago Genomics Facility where RNA-seq library preparation was carried out. RNA libraries were sequenced using an Illumina HiSeq 4000 platform (1 x 50 bp single end sequencing). RNA sequencing files were transferred to the Tarbell High-performance computing cluster of Center for Research Informatics at the University of Chicago for analysis. The quality of raw sequencing data was assessed using FastQC v0.11.5. All RNA reads were first mapped to the mouse reference genome $(\mathrm{mm} 10)$ using STAR v2.5.2b release with default parameters. Picard v2.8.1 (http://broadinstitute.github.io/picard/) was used to collect mapping metrics. Reads were mapped to the mm10 mouse genome assembly. The counted reads were filtered to exclude reads with identical library- and molecule barcodes. Differential gene expression analysis was performed using the DESeq2 package. Significance was defined as FDR corrected p-value $<$ 0.05 and absolute Fold Change $\geq 1.5$.

\section{0-plex Tandem Mass Tags (TMT) Proteomic Analysis}

Five Brap ${ }^{\text {cKONPC }}$ and five control (Brap ${ }^{\text {flox/floxCre- }}$ or Brap ${ }^{\text {flox/WTCre- }}$ ) mice at 6 months of age were transcardially perfused with ice cold PBS and protease inhibitors to remove high-abundant blood and serum proteins. Cerebral cortical tissue was dissected on ice, flash frozen in liquid nitrogen, and sent to the Thermo Fisher Center of Harvard Medical School where the samples were processed for 10-plex TMT analysis. MS2 spectra were searched using the SEQUEST algorithm against a Uniprot composite database derived from Mouse proteome containing its reversed complement and known contaminants. Peptide spectral matches were filtered to a $1 \%$ false discovery rate (FDR) using the target-decoy strategy combined with linear discriminant analysis. 
The proteins were filtered to a $<1 \%$ FDR. Proteins were quantified only from peptides with a summed SN threshold of $>100$ and MS2 isolation specificity of 0.5 .

\section{Quantification and Statistical Analysis}

No statistical methods were used to predetermine sample size, while all experiments were performed with a minimum of three biological replicates and all cell counts were obtained from at least ten random fields. The experiments were not randomized; the investigators were not blinded to the sample allocation and data acquisition during experiments but were blinded in performing quantitative analyses of immunohistological images using the NIH Image J software.

All statistical analyses were done using GraphPad Prism 7.0 software. Data were analyzed by one-way ANOVA or unpaired two-tailed Student's t tests for comparing differences between different genotypes. Differences were considered significant with a $p$ value $<0.05$.

\section{ACKNOWLEDGMENTS}

The authors wish to thank Barrington Burnett of Uniformed Services University for communication and discussion; the Genomics Facility of University of Chicago for RNA library construction and NGS analysis; the Thermo Fisher Center for Multiplexed Proteomics at the Harvard Medical School for tandem mass tag proteomic analysis; and the Biomedical Instrumentation Center of Uniformed Services University for qRT-PCR analysis. This work was supported by startup funds of Northwestern University and USUHS to Y.F. The UCI ADRC is funded by NIH/NIA Grant P30AG066519.

\section{AUTHOR CONTRIBUTIONS:}


Y.F. conceptualized the project, designed and performed the experiments, interpreted the results, and wrote the manuscript. Y.G. performed experiments; A.A.C. initiated the study and performed the experiments. Y.H. performed data analyses, C.C.L performed experiments, H.P. performed experiments. W-C.C. and J.A. performed bioinformatics data processing and analysis. X.Z. assisted with experiments. E.B. assisted with bioinformatics data analysis. E.S.M. helped with brain pathology data interpretation and the acquisition of the human $\mathrm{AD}$ and control brain specimens from the UCI ADRC.

COMPETING INTERESTS: The authors declare that they have no conflict of interest.

\section{REFERENCES}

Aoshiba, K., Tsuji, T., Kameyama, S., Itoh, M., Semba, S., Yamaguchi, K., and Nakamura, H. (2013). Senescence-associated secretory phenotype in a mouse model of bleomycin-induced lung injury. Exp Toxicol Pathol 65, 1053-1062.

Bushman, D.M., Kaeser, G.E., Siddoway, B., Westra, J.W., Rivera, R.R., Rehen, S.K., Yung, Y.C., and Chun, J. (2015). Genomic mosaicism with increased amyloid precursor protein (APP) gene copy number in single neurons from sporadic Alzheimer's disease brains. Elife 4.

Campisi, J., and Robert, L. (2014). Cell senescence: role in aging and age-related diseases. Interdiscip Top Gerontol 39, 45-61.

Cao, L., Li, W., Kim, S., Brodie, S.G., and Deng, C.X. (2003). Senescence, aging, and malignant transformation mediated by p53 in mice lacking the Brca1 full-length isoform. Genes Dev 17, 201-213.

Capparelli, C., Chiavarina, B., Whitaker-Menezes, D., Pestell, T.G., Pestell, R.G., Hulit, J., Ando, S., Howell, A., Martinez-Outschoorn, U.E., Sotgia, F., et al. (2012). CDK inhibitors (p16/p19/p21) induce senescence and autophagy in cancer-associated fibroblasts, "fueling" tumor growth via paracrine interactions, without an increase in neo-angiogenesis. Cell Cycle 11, 3599-3610. 
Castello, L.M., Raineri, D., Salmi, L., Clemente, N., Vaschetto, R., Quaglia, M., Garzaro, M., Gentilli, S., Navalesi, P., Cantaluppi, V., et al. (2017). Osteopontin at the Crossroads of Inflammation and Tumor Progression. Mediators Inflamm 2017, 4049098.

Chen, Y., Chen, C.F., Riley, D.J., Allred, D.C., Chen, P.L., Von Hoff, D., Osborne, C.K., and Lee, W.H. (1995). Aberrant subcellular localization of BRCA1 in breast cancer. Science 270, 789-791.

Choy, K.R., and Watters, D.J. (2018). Neurodegeneration in ataxia-telangiectasia: Multiple roles of ATM kinase in cellular homeostasis. Dev Dyn 247, 33-46.

Coppe, J.P., Desprez, P.Y., Krtolica, A., and Campisi, J. (2010). The senescence-associated secretory phenotype: the dark side of tumor suppression. Annu Rev Pathol 5, 99-118.

Coppe, J.P., Patil, C.K., Rodier, F., Sun, Y., Munoz, D.P., Goldstein, J., Nelson, P.S., Desprez, P.Y., and Campisi, J. (2008). Senescence-associated secretory phenotypes reveal cellnonautonomous functions of oncogenic RAS and the p53 tumor suppressor. PLoS Biol 6, 28532868.

Coppede, F., and Migliore, L. (2010). DNA repair in premature aging disorders and neurodegeneration. Curr Aging Sci 3, 3-19.

Dewachter, I., Reverse, D., Caluwaerts, N., Ris, L., Kuiperi, C., Van den Haute, C., Spittaels, K., Umans, L., Serneels, L., Thiry, E., et al. (2002). Neuronal deficiency of presenilin 1 inhibits amyloid plaque formation and corrects hippocampal long-term potentiation but not a cognitive defect of amyloid precursor protein [V717I] transgenic mice. J Neurosci 22, 3445-3453.

Dou, Z., Xu, C., Donahue, G., Shimi, T., Pan, J.A., Zhu, J., Ivanov, A., Capell, B.C., Drake, A.M., Shah, P.P., et al. (2015). Autophagy mediates degradation of nuclear lamina. Nature 527, 105-109.

Flanagan, K.C., Alspach, E., Pazolli, E., Parajuli, S., Ren, Q., Arthur, L.L., Tapia, R., and Stewart, S.A. (2018). c-Myb and C/EBPbeta regulate OPN and other senescence-associated secretory phenotype factors. Oncotarget 9, 21-36.

Freund, A., Orjalo, A.V., Desprez, P.Y., and Campisi, J. (2010). Inflammatory networks during cellular senescence: causes and consequences. Trends Mol Med 16, 238-246.

Frost, G.R., Jonas, L.A., and Li, Y.M. (2019). Friend, Foe or Both? Immune Activity in Alzheimer's Disease. Front Aging Neurosci 11, 337.

Ghosh, K., and Capell, B.C. (2016). The Senescence-Associated Secretory Phenotype: Critical Effector in Skin Cancer and Aging. J Invest Dermatol 136, 2133-2139. 
Gorski, J.A., Talley, T., Qiu, M., Puelles, L., Rubenstein, J.L., and Jones, K.R. (2002). Cortical excitatory neurons and glia, but not GABAergic neurons, are produced in the Emx1-expressing lineage. J Neurosci 22, 6309-6314.

Govin, J., Caron, C., Lestrat, C., Rousseaux, S., and Khochbin, S. (2004). The role of histones in chromatin remodelling during mammalian spermiogenesis. Eur J Biochem 271, 3459-3469.

Haber, D.A. (1997). Splicing into senescence: the curious case of p16 and p19ARF. Cell 91, 555-558.

Hayflick, L. (1965). The Limited in Vitro Lifetime of Human Diploid Cell Strains. Exp Cell Res 37, 614-636.

Hernandez-Segura, A., Nehme, J., and Demaria, M. (2018). Hallmarks of Cellular Senescence. Trends Cell Biol 28, 436-453.

Hoang, M.L., Kinde, I., Tomasetti, C., McMahon, K.W., Rosenquist, T.A., Grollman, A.P., Kinzler, K.W., Vogelstein, B., and Papadopoulos, N. (2016). Genome-wide quantification of rare somatic mutations in normal human tissues using massively parallel sequencing. Proc Natl Acad Sci U S A 113, 9846-9851.

Horn, V., Uckelmann, M., Zhang, H., Eerland, J., Aarsman, I., le Paige, U.B., Davidovich, C., Sixma, T.K., and van Ingen, H. (2019). Structural basis of specific H2A K13/K15 ubiquitination by RNF168. Nat Commun 10, 1751.

Houlihan, S.L., and Feng, Y. (2014). The scaffold protein Nde1 safeguards the brain genome during $\mathrm{S}$ phase of early neural progenitor differentiation. Elife 3, e03297.

Ivanov, A., Pawlikowski, J., Manoharan, I., van Tuyn, J., Nelson, D.M., Rai, T.S., Shah, P.P., Hewitt, G., Korolchuk, V.I., Passos, J.F., et al. (2013). Lysosome-mediated processing of chromatin in senescence. The Journal of cell biology 202, 129-143.

Kakigi, R., Endo, C., Neshige, R., Kohno, H., and Kuroda, Y. (1992). Accelerated aging of the brain in Werner's syndrome. Neurology 42, 922-924.

Kalb, R., Mallery, D.L., Larkin, C., Huang, J.T., and Hiom, K. (2014). BRCA1 is a histoneH2A-specific ubiquitin ligase. Cell reports 8, 999-1005.

Kim, B.J., Chan, D.W., Jung, S.Y., Chen, Y., Qin, J., and Wang, Y. (2017). The Histone Variant MacroH2A1 Is a BRCA1 Ubiquitin Ligase Substrate. Cell reports 19, 1758-1766.

Kim, B.W., Jeong, Y.E., Wong, M., and Martin, L.J. (2020). DNA damage accumulates and responses are engaged in human ALS brain and spinal motor neurons and DNA repair is activatable in iPSC-derived motor neurons with SOD1 mutations. Acta Neuropathol Commun 8, 7. 
Kuilman, T., and Peeper, D.S. (2009). Senescence-messaging secretome: SMS-ing cellular stress. Nat Rev Cancer 9, 81-94.

Lanctot, A.A., Guo, Y., Le, Y., Edens, B.M., Nowakowski, R.S., and Feng, Y. (2017). Loss of Brap Results in Premature G1/S Phase Transition and Impeded Neural Progenitor Differentiation. Cell reports 20, 1148-1160.

Lanctot, A.A., Peng, C.Y., Pawlisz, A.S., Joksimovic, M., and Feng, Y. (2013). Spatially dependent dynamic MAPK modulation by the Nde1-Lis1-Brap complex patterns mammalian CNS. Dev Cell 25, 241-255.

Lasry, A., and Ben-Neriah, Y. (2015). Senescence-associated inflammatory responses: aging and cancer perspectives. Trends Immunol 36, 217-228.

Li, S., Ku, C.Y., Farmer, A.A., Cong, Y.S., Chen, C.F., and Lee, W.H. (1998). Identification of a novel cytoplasmic protein that specifically binds to nuclear localization signal motifs. J Biol Chem 273, 6183-6189.

Lodato, M.A., Rodin, R.E., Bohrson, C.L., Coulter, M.E., Barton, A.R., Kwon, M., Sherman, M.A., Vitzthum, C.M., Luquette, L.J., Yandava, C.N., et al. (2018). Aging and neurodegeneration are associated with increased mutations in single human neurons. Science 359, 555-559.

Lodato, M.A., Woodworth, M.B., Lee, S., Evrony, G.D., Mehta, B.K., Karger, A., Lee, S., Chittenden, T.W., D'Gama, A.M., Cai, X., et al. (2015). Somatic mutation in single human neurons tracks developmental and transcriptional history. Science 350, 94-98.

Lombard, D.B., Chua, K.F., Mostoslavsky, R., Franco, S., Gostissa, M., and Alt, F.W. (2005). DNA repair, genome stability, and aging. Cell 120, 497-512.

Lopez-Otin, C., Blasco, M.A., Partridge, L., Serrano, M., and Kroemer, G. (2013). The hallmarks of aging. Cell 153, 1194-1217.

Lu, T., Pan, Y., Kao, S.Y., Li, C., Kohane, I., Chan, J., and Yankner, B.A. (2004). Gene regulation and DNA damage in the ageing human brain. Nature 429, 883-891.

Luczak, M.W., and Zhitkovich, A. (2018). Monoubiquitinated gamma-H2AX: Abundant product and specific biomarker for non-apoptotic DNA double-strand breaks. Toxicol Appl Pharmacol 355, 238-246.

Madabhushi, R., Pan, L., and Tsai, L.H. (2014). DNA damage and its links to neurodegeneration. Neuron 83, 266-282.

Matheny, S.A., Chen, C., Kortum, R.L., Razidlo, G.L., Lewis, R.E., and White, M.A. (2004). Ras regulates assembly of mitogenic signalling complexes through the effector protein IMP. Nature 427, 256-260. 
Mattson, M.P., and Arumugam, T.V. (2018). Hallmarks of Brain Aging: Adaptive and Pathological Modification by Metabolic States. Cell Metab 27, 1176-1199.

McHugh, D., and Gil, J. (2018). Senescence and aging: Causes, consequences, and therapeutic avenues. The Journal of cell biology 217, 65-77.

Mitra, J., Guerrero, E.N., Hegde, P.M., Liachko, N.F., Wang, H., Vasquez, V., Gao, J., Pandey, A., Taylor, J.P., Kraemer, B.C., et al. (2019). Motor neuron disease-associated loss of nuclear TDP-43 is linked to DNA double-strand break repair defects. Proc Natl Acad Sci U S A 116, 4696-4705.

Munoz-Espin, D., and Serrano, M. (2014). Cellular senescence: from physiology to pathology. Nat Rev Mol Cell Biol 15, 482-496.

Newcombe, E.A., Camats-Perna, J., Silva, M.L., Valmas, N., Huat, T.J., and Medeiros, R. (2018). Inflammation: the link between comorbidities, genetics, and Alzheimer's disease. J Neuroinflammation 15, 276.

Padeken, J., Zeller, P., Towbin, B., Katic, I., Kalck, V., Methot, S.P., and Gasser, S.M. (2019). Synergistic lethality between BRCA1 and H3K9me2 loss reflects satellite derepression. Genes Dev 33, 436-451.

Panier, S., and Boulton, S.J. (2014). Double-strand break repair: 53BP1 comes into focus. Nat Rev Mol Cell Biol 15, 7-18.

Pazolli, E., Alspach, E., Milczarek, A., Prior, J., Piwnica-Worms, D., and Stewart, S.A. (2012). Chromatin remodeling underlies the senescence-associated secretory phenotype of tumor stromal fibroblasts that supports cancer progression. Cancer Res 72, 2251-2261.

Pulvers, J.N., and Huttner, W.B. (2009). Brca1 is required for embryonic development of the mouse cerebral cortex to normal size by preventing apoptosis of early neural progenitors. Development 136, 1859-1868.

Quelle, D.E., Zindy, F., Ashmun, R.A., and Sherr, C.J. (1995). Alternative reading frames of the INK4a tumor suppressor gene encode two unrelated proteins capable of inducing cell cycle arrest. Cell 83, 993-1000.

Rao, S.G., and Jackson, J.G. (2016). SASP: Tumor Suppressor or Promoter? Yes! Trends Cancer 2, 676-687.

Reid, L.J., Shakya, R., Modi, A.P., Lokshin, M., Cheng, J.T., Jasin, M., Baer, R., and Ludwig, T. (2008). E3 ligase activity of BRCA1 is not essential for mammalian cell viability or homologydirected repair of double-strand DNA breaks. Proc Natl Acad Sci U S A 105, 20876-20881. 
Rodier, F., and Campisi, J. (2011). Four faces of cellular senescence. The Journal of cell biology $192,547-556$.

Rulten, S.L., and Caldecott, K.W. (2013). DNA strand break repair and neurodegeneration. DNA Repair (Amst) 12, 558-567.

Rutten, B.P., Schmitz, C., Gerlach, O.H., Oyen, H.M., de Mesquita, E.B., Steinbusch, H.W., and Korr, H. (2007). The aging brain: accumulation of DNA damage or neuron loss? Neurobiol Aging 28, 91-98.

Salminen, A., Kauppinen, A., and Kaarniranta, K. (2012). Emerging role of NF-kappaB signaling in the induction of senescence-associated secretory phenotype (SASP). Cell Signal 24, 835-845.

Sarlus, H., and Heneka, M.T. (2017). Microglia in Alzheimer's disease. The Journal of clinical investigation 127, 3240-3249.

Schaser, A.J., Osterberg, V.R., Dent, S.E., Stackhouse, T.L., Wakeham, C.M., Boutros, S.W., Weston, L.J., Owen, N., Weissman, T.A., Luna, E., et al. (2019). Alpha-synuclein is a DNA binding protein that modulates DNA repair with implications for Lewy body disorders. Scientific reports 9, 10919.

Scully, R., Ganesan, S., Brown, M., De Caprio, J.A., Cannistra, S.A., Feunteun, J., Schnitt, S., and Livingston, D.M. (1996). Location of BRCA1 in human breast and ovarian cancer cells. Science 272, 123-126.

Shanbhag, N.M., Evans, M.D., Mao, W., Nana, A.L., Seeley, W.W., Adame, A., Rissman, R.A., Masliah, E., and Mucke, L. (2019). Early neuronal accumulation of DNA double strand breaks in Alzheimer's disease. Acta Neuropathol Commun 7, 77.

Soares, J.P., Cortinhas, A., Bento, T., Leitao, J.C., Collins, A.R., Gaivao, I., and Mota, M.P. (2014). Aging and DNA damage in humans: a meta-analysis study. Aging (Albany NY) 6, 432439.

Suberbielle, E., Sanchez, P.E., Kravitz, A.V., Wang, X., Ho, K., Eilertson, K., Devidze, N., Kreitzer, A.C., and Mucke, L. (2013). Physiologic brain activity causes DNA double-strand breaks in neurons, with exacerbation by amyloid-beta. Nat Neurosci 16, 613-621.

Tamburri, S., Lavarone, E., Fernandez-Perez, D., Conway, E., Zanotti, M., Manganaro, D., and Pasini, D. (2020). Histone H2AK119 Mono-Ubiquitination Is Essential for Polycomb-Mediated Transcriptional Repression. Mol Cell 77, 840-856 e845.

Thadathil, N., Hori, R., Xiao, J., and Khan, M.M. (2019). DNA double-strand breaks: a potential therapeutic target for neurodegenerative diseases. Chromosome Res 27, 345-364. 
Thiriet, C., and Hayes, J.J. (2005). Chromatin in need of a fix: phosphorylation of H2AX connects chromatin to DNA repair. Mol Cell 18, 617-622.

Timmers, P.R., Mounier, N., Lall, K., Fischer, K., Ning, Z., Feng, X., Bretherick, A.D., Clark, D.W., e, Q.C., Agbessi, M., et al. (2019). Genomics of 1 million parent lifespans implicates novel pathways and common diseases and distinguishes survival chances. Elife 8.

Todaro, G.J., and Green, H. (1963). Quantitative studies of the growth of mouse embryo cells in culture and their development into established lines. The Journal of cell biology 17, 299-313.

Uckelmann, M., and Sixma, T.K. (2017). Histone ubiquitination in the DNA damage response. DNA Repair (Amst) 56, 92-101.

Vaughan, D.E., Rai, R., Khan, S.S., Eren, M., and Ghosh, A.K. (2017). Plasminogen Activator Inhibitor-1 Is a Marker and a Mediator of Senescence. Arterioscler Thromb Vasc Biol 37, 14461452.

Wang, H., Wang, L., Erdjument-Bromage, H., Vidal, M., Tempst, P., Jones, R.S., and Zhang, Y. (2004). Role of histone H2A ubiquitination in Polycomb silencing. Nature 431, 873-878.

Weidenheim, K.M., Dickson, D.W., and Rapin, I. (2009). Neuropathology of Cockayne syndrome: Evidence for impaired development, premature aging, and neurodegeneration. Mech Ageing Dev 130, 619-636.

White, R.R., and Vijg, J. (2016). Do DNA Double-Strand Breaks Drive Aging? Mol Cell 63, 729-738.

Wilson, C.A., Ramos, L., Villasenor, M.R., Anders, K.H., Press, M.F., Clarke, K., Karlan, B., Chen, J.J., Scully, R., Livingston, D., et al. (1999). Localization of human BRCA1 and its loss in high-grade, non-inherited breast carcinomas. Nat Genet 21, 236-240.

Wu, J., Huen, M.S., Lu, L.Y., Ye, L., Dou, Y., Ljungman, M., Chen, J., and Yu, X. (2009). Histone ubiquitination associates with BRCA1-dependent DNA damage response. Mol Cell Biol 29, 849-860.

Wu, W., Koike, A., Takeshita, T., and Ohta, T. (2008). The ubiquitin E3 ligase activity of BRCA1 and its biological functions. Cell Div 3, 1.

Yang, L., Ma, Z., Wang, H., Niu, K., Cao, Y., Sun, L., Geng, Y., Yang, B., Gao, F., Chen, Z., et al. (2019). Ubiquitylome study identifies increased histone 2A ubiquitylation as an evolutionarily conserved aging biomarker. Nat Commun 10, 2191.

Zhu, Q., Pao, G.M., Huynh, A.M., Suh, H., Tonnu, N., Nederlof, P.M., Gage, F.H., and Verma, I.M. (2011). BRCA1 tumour suppression occurs via heterochromatin-mediated silencing. Nature $477,179-184$. 
A
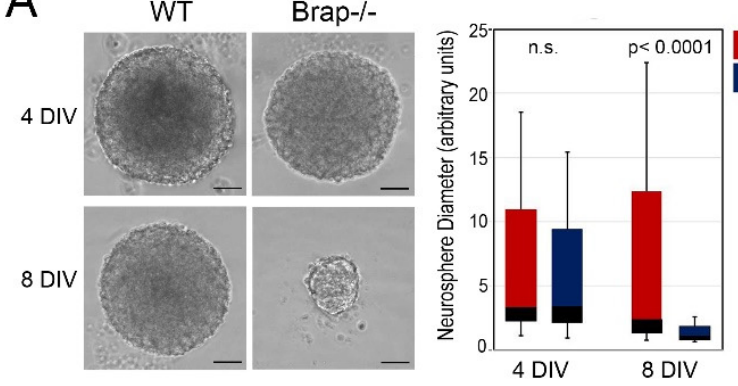

C

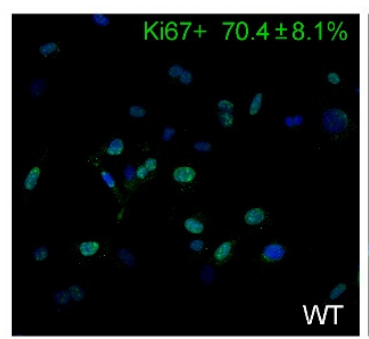

D

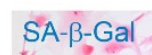

WT

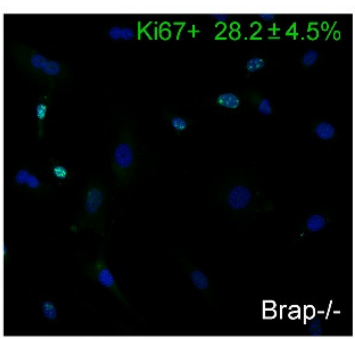

SA- $\beta-G a l$

G
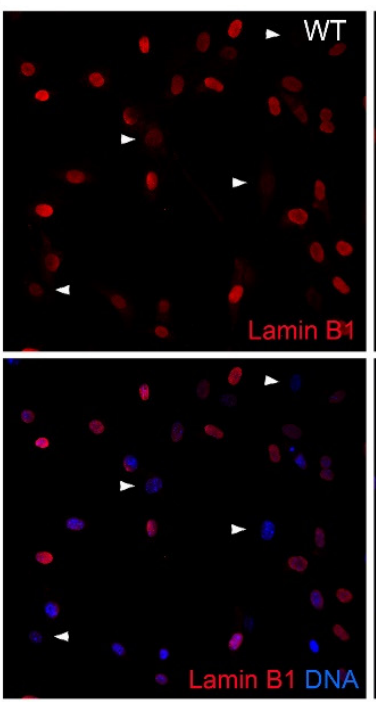

B

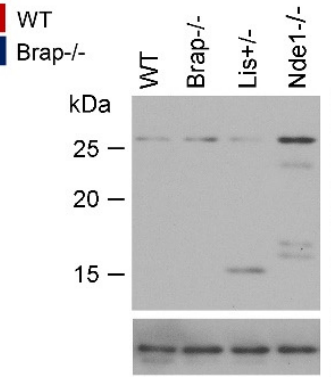

NPC
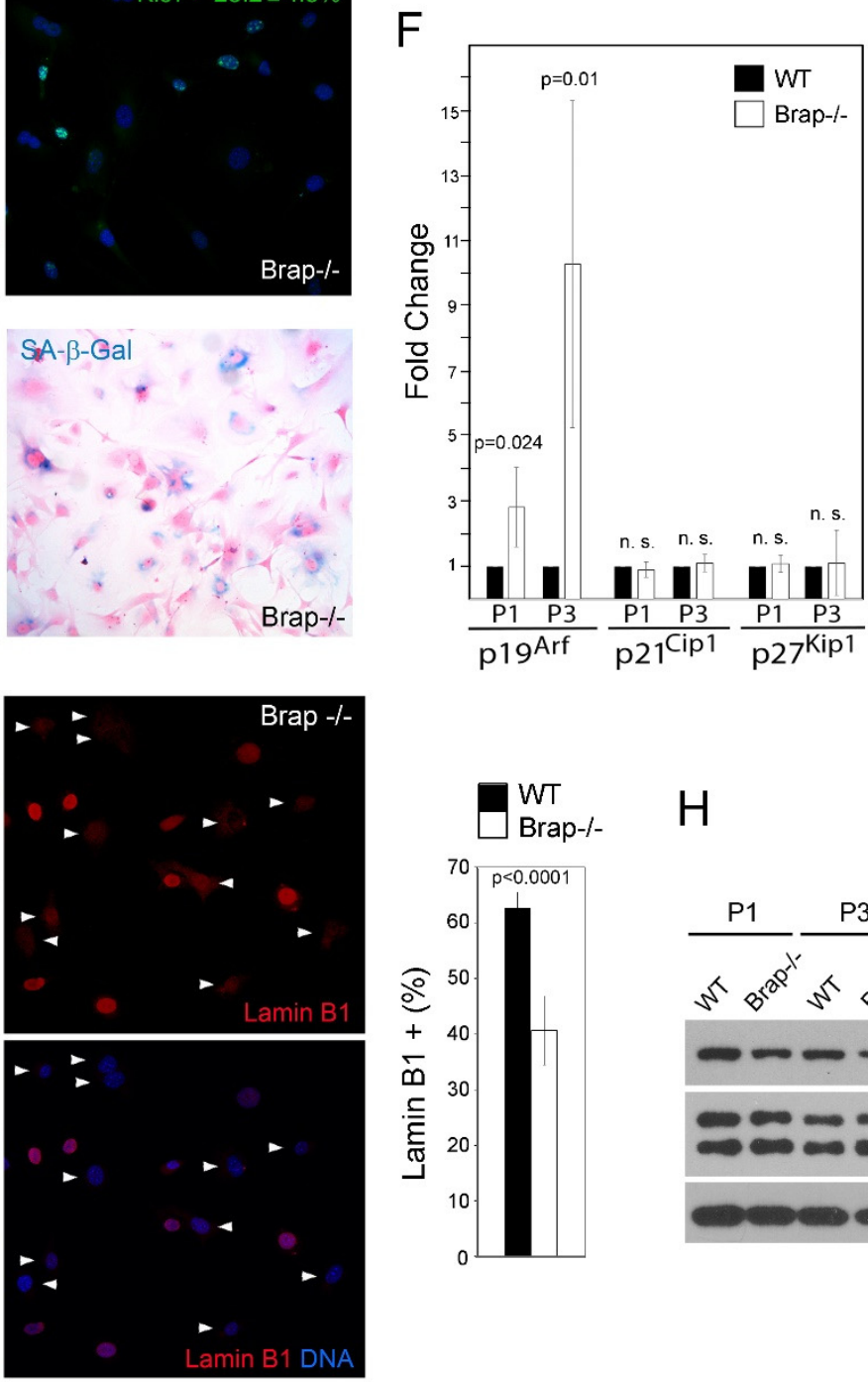

E

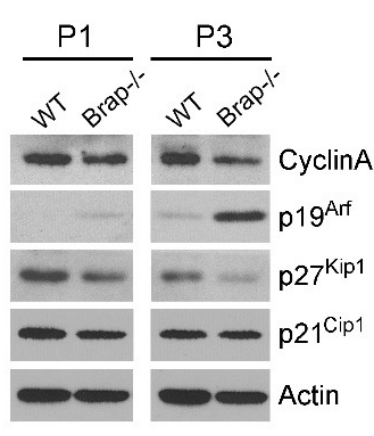

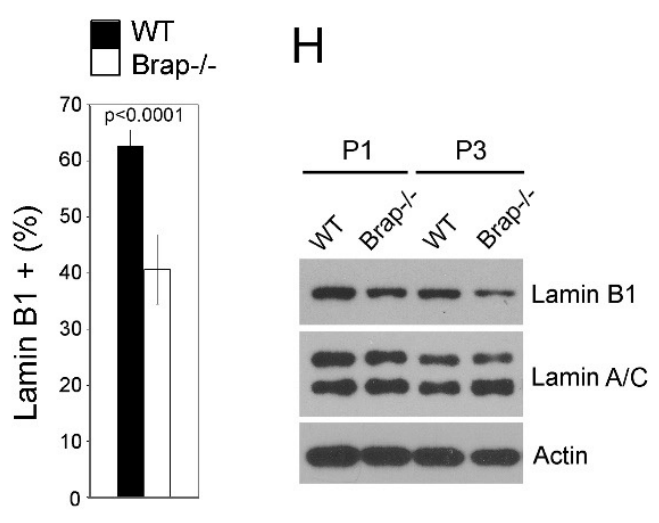




\section{FIGURE 1. BRAP LOSS OF FUNCTION (LOF) RESULTS IN ACCELERATED CELLULAR SENESCENCE.}

A. Primary Brap ${ }^{-/-}$neural progenitor cells (NPC) cease growing in culture after 5-7 days in vitro (DIV). Shown are representative images of WT and Brap ${ }^{-/}$neurospheres as well as box and whisker plots (Median $\pm \mathrm{SD}$ ) of their diameter distribution at 4 DIV and 8 DIV, respectively.

B. Cleaved caspase 3 (CC3) immunoblotting of total protein extracts of primary NPCs and MEFs, respectively. $\mathrm{Lis}^{+/-}$and $\mathrm{Nde}^{-/-}$cells were used for CC3+ control as these cells were known to have high levels of apoptosis. C. Representative Ki67 immunofluorescence images of WT and Brap ${ }^{-/-}$MEFs at P2. The \% of Ki67+ cells $($ Mean \pm SD) is indicated.

D. Senescence-associated $\beta$-gal assay of MEFs at P2, which indicates $\beta$-galactosidase activity (blue) in higher fraction of Brap ${ }^{-/}$cells. Cells were also counter-stained with eosin to view cell density and cell shape. Note the enlarged cell body of $\beta$-gal+ cells.

$\mathrm{E}, \mathrm{F}$. Immunoblotting and quantification (Mean $\pm \mathrm{SD}$ ) of G1/S CKIs in total protein extracts from MEFs at P1 and P3, respectively.

G. Representative Lamin B1 immunofluorescence images and percentage (Mean \pm SD) of Lamin B1+ MEFs at P2. Cells showing significant reduction of nuclear Lamin B1 are indicated by arrowheads.

H. Immunoblotting of total protein extracts, showing reduced Lamin B1 in Brap ${ }^{-/-}$relative to WT MEFs at both P1 and P3.

I. Immunoblotting of total protein extracts from MEFs at P1 and P3, showing increased SASP molecules in Brap $^{-/-}$MEFs at P3. Note the increased Mmps and decreased Mmp substrate fibronection (FN) in Brap ${ }^{-/-}$MEFs.

Nuclear DNA was stained with Hoechst 33342. Bars: 100 um or as indicated. 
bioRxiv preprint doi: https://doi.org/10.1101/2020.10.22.341784; this version posted January 9, 2021. The copyright holder for this preprint (which was not certified by peer review) is the author/funder. All rights reserved. No reuse allowed without permission.

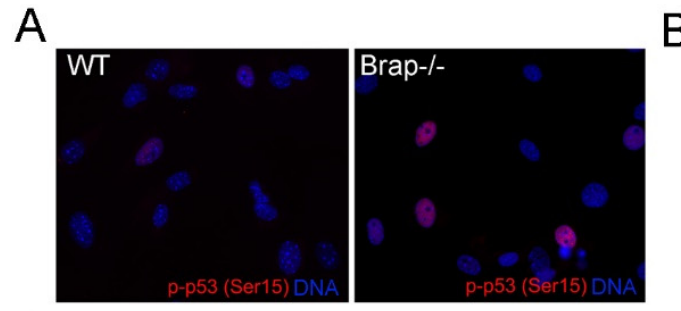

D

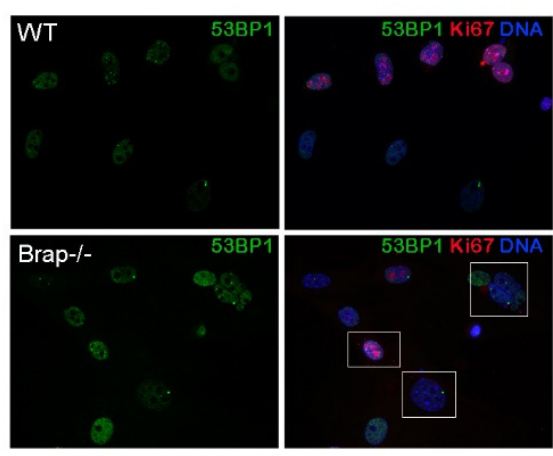

G

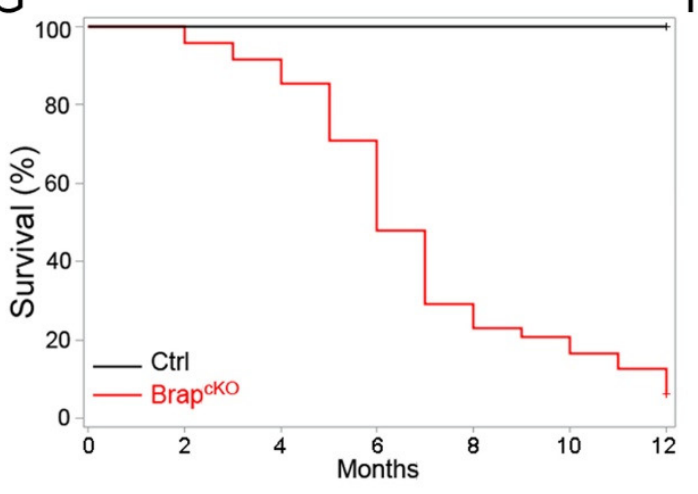

B

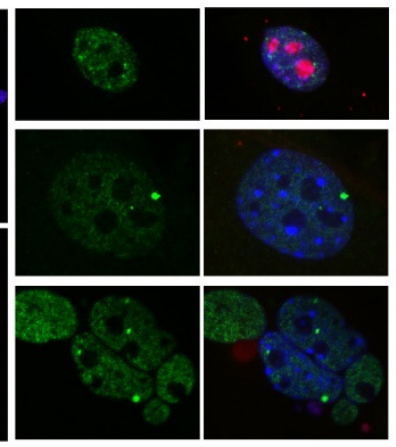

$\mathrm{H}$
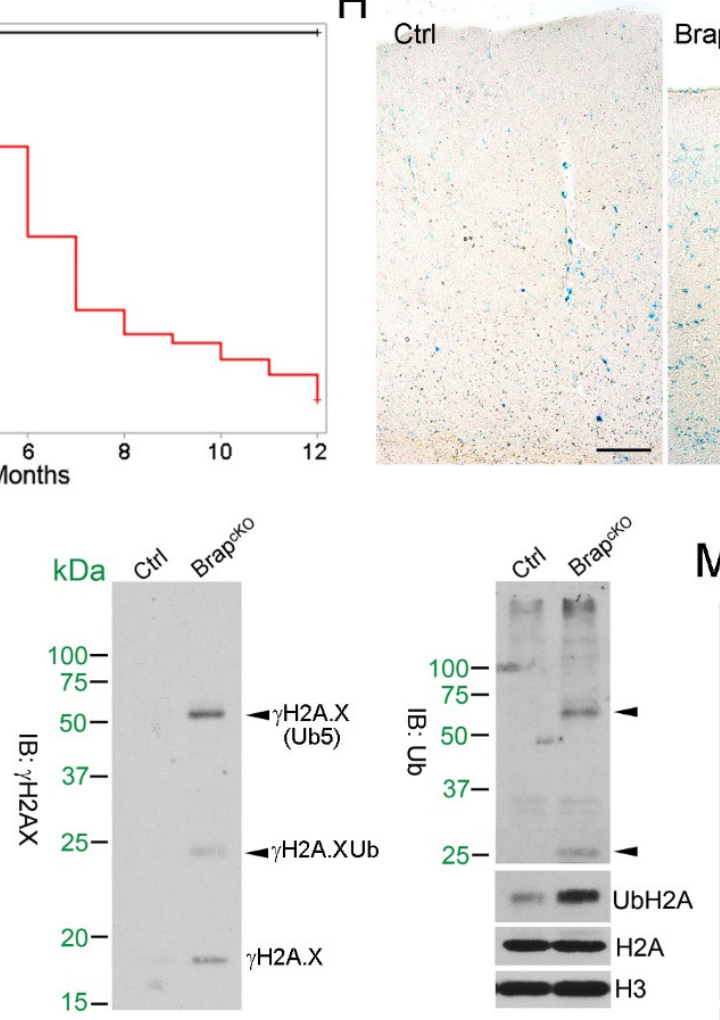

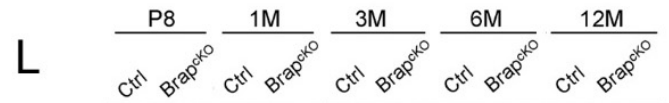

K

$\mathrm{N}$

M
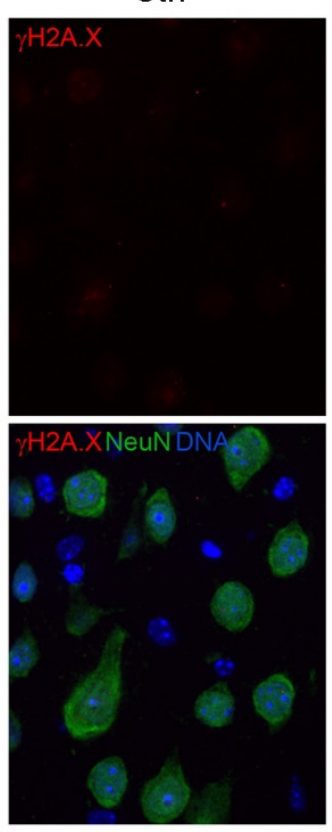

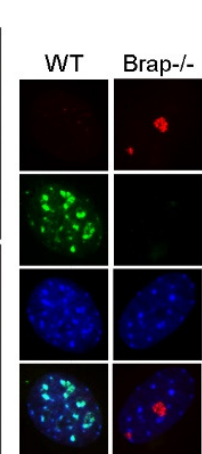

BrapcKO
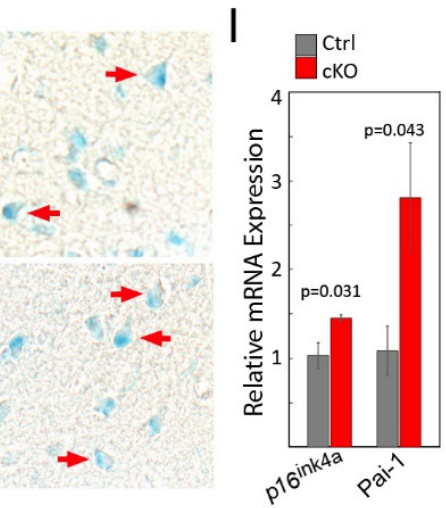

J
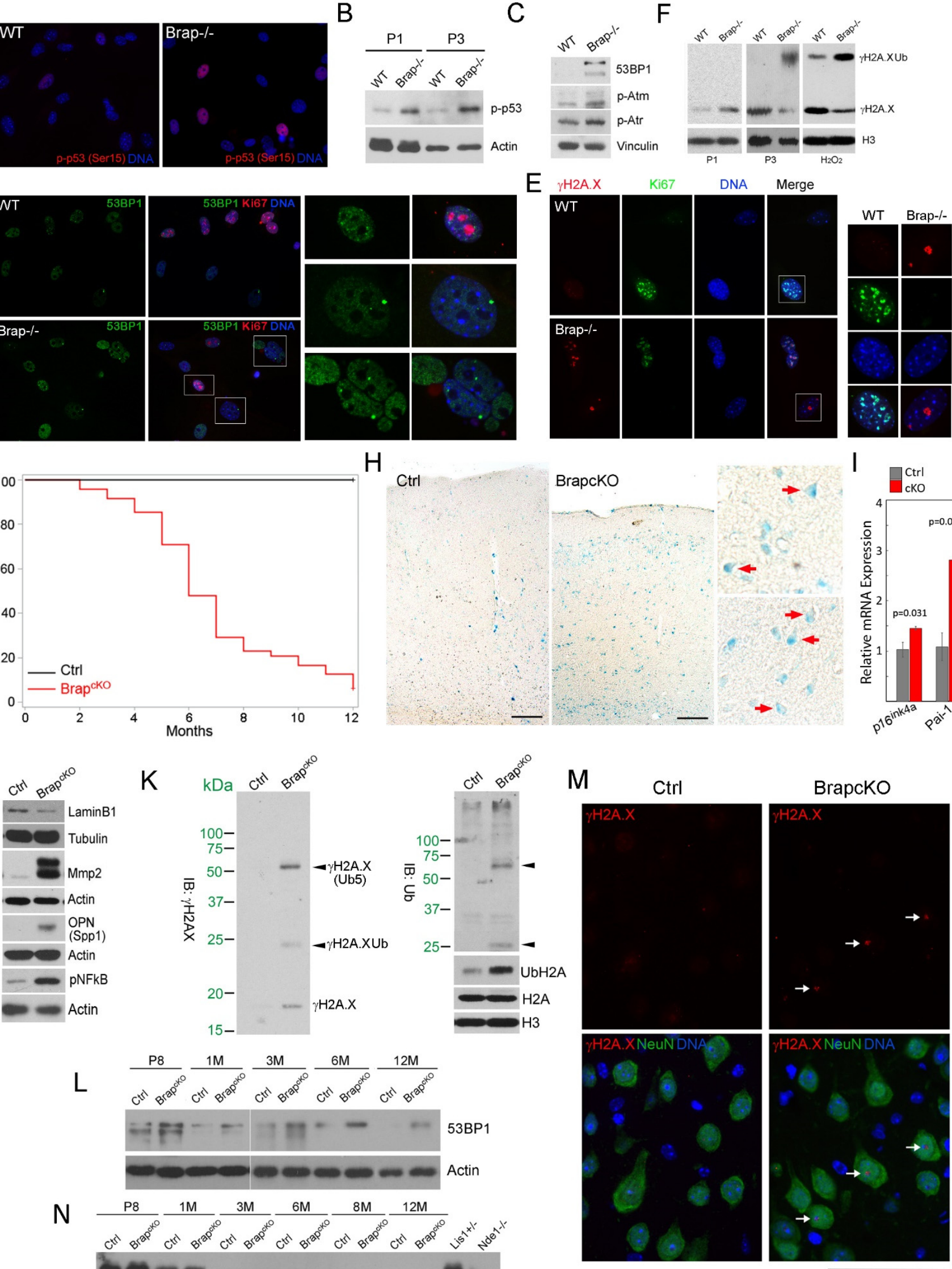


\section{FIGURE 2. INCREASED DSB AND DDR ARE ASSOCIATED WITH CELLULAR SENESCENCE IN BRAP DEFICIENT CELLS AND CEREBRAL CORTICES.}

A. Representative images of phospho-p53 immunofluorescence staining of WT and Brap ${ }^{-/-}$MEFs at P2.

B. Immunoblotting of total protein extracts from WT and Brap ${ }^{-/-} \mathrm{MEFs}$ at $\mathrm{P} 1$ and $\mathrm{P} 3$, respectively.

C. Immunoblotting of DNA damage response (DDR) proteins in WT and Brap ${ }^{-/-} \mathrm{MEFs}$ at P1.

D. Representative images of double immunofluorescence staining of WT and Brap ${ }^{-/-}$MEFs at P3 with antibodies against Ki67 (red) and 53BP1 (green).

E. Representative images of double immunofluorescence staining of WT and Brap ${ }^{-/-}$MEFs at P3 with antibodies against Ki67 (green) and $\gamma \mathrm{H} 2 \mathrm{~A} . \mathrm{X}$ (red).

F. Immunoblotting analyses of histone extracts, showing increased $\gamma \mathrm{H} 2 \mathrm{~A}$.X and $\gamma \mathrm{H} 2 \mathrm{~A}$.X mono-ubiquitination in Brap $^{-/-}$MEFs.

G. Kaplan-Meier curve shows significantly shortened lifespan of Brap ${ }^{\text {cKONPC }}$ mice $(n=46)$ relative to their littermate control mice $(\mathrm{n}=29)$.

H. Representative images of senescence-associated $\beta$-gal analysis of cerebral cortical sections from Brap ${ }^{\mathrm{cKONPC}}$ and control mice at 3 months of age. Note the pyramidal neuronal morphology of some SA- $\beta$ gal+ cells (red arrows) in high magnification views.

I. RT-qPCR shows increased expression of $\mathrm{p} 16^{\text {Ink4a }}$ and Pai-1 (plasminogen activator inhibitor-1/Serpine1) in 3month old Brap ${ }^{\text {cKONPC }}$ cortices (Mean $\pm \mathrm{SD} ; \mathrm{n}=4$ biological replicates).

J. Immunoblotting of cerebral cortical total protein extracts, demonstrating higher levels of senescence and SASP-like changes in the cortical tissue of Brap ${ }^{\text {cKONPC }}$ relative to littermate control mice.

K. Immunoblotting of histone extracts from cortical tissue of 3-month old Brap ${ }^{\mathrm{cKONPC}}$ and control mice, showing elevated $\gamma \mathrm{H} 2 \mathrm{~A} . \mathrm{X}$ as well as the presence of mono- and poly- $\gamma \mathrm{H} 2 \mathrm{~A} . \mathrm{X}$ ubiquitination in Brap ${ }^{\mathrm{cKONPC}}$ mice.

L. Immunoblotting of total protein extracts from cortical tissue of Brap ${ }^{\text {cKONPC }}$ and control mice, showing persistent elevation of 53BP1 in Brap ${ }^{\text {cKONPC }}$ cortices.

M. Representative images of $\gamma \mathrm{H} 2 \mathrm{~A}$.X-NeuN double immunohistological staining of cerebral cortical sections from 4-month old WT or Brap ${ }^{\mathrm{cKONPC}}$ mice. The presence of $\gamma \mathrm{H} 2 \mathrm{~A}$.X immunoreactivity in Brap ${ }^{\mathrm{cKONPC}}$ neurons are indicated by arrows.

N. Immunoblotting of total protein extracts from cortical tissue of Brap ${ }^{\text {cKONPC }}$ or control mice at various ages, showing that Brap LOF does not increase cleaved caspase 3, an apoptosis marker. Embryonic Lis $1^{+/}$and $\mathrm{Nde}^{-/-}$cortical tissues were used as positive controls for the presence of apoptosis.

Nuclear DNA was stained with Hoechst 33342. Bars: 100 um or as indicated. 
bioRxiv preprint doi: https://doi.org/10.1101/2020.10.22.341784; this version posted January 9, 2021. The copyright holder for this preprint (which was not certified by peer review) is the author/funder. All rights reserved. No reuse allowed without permission.

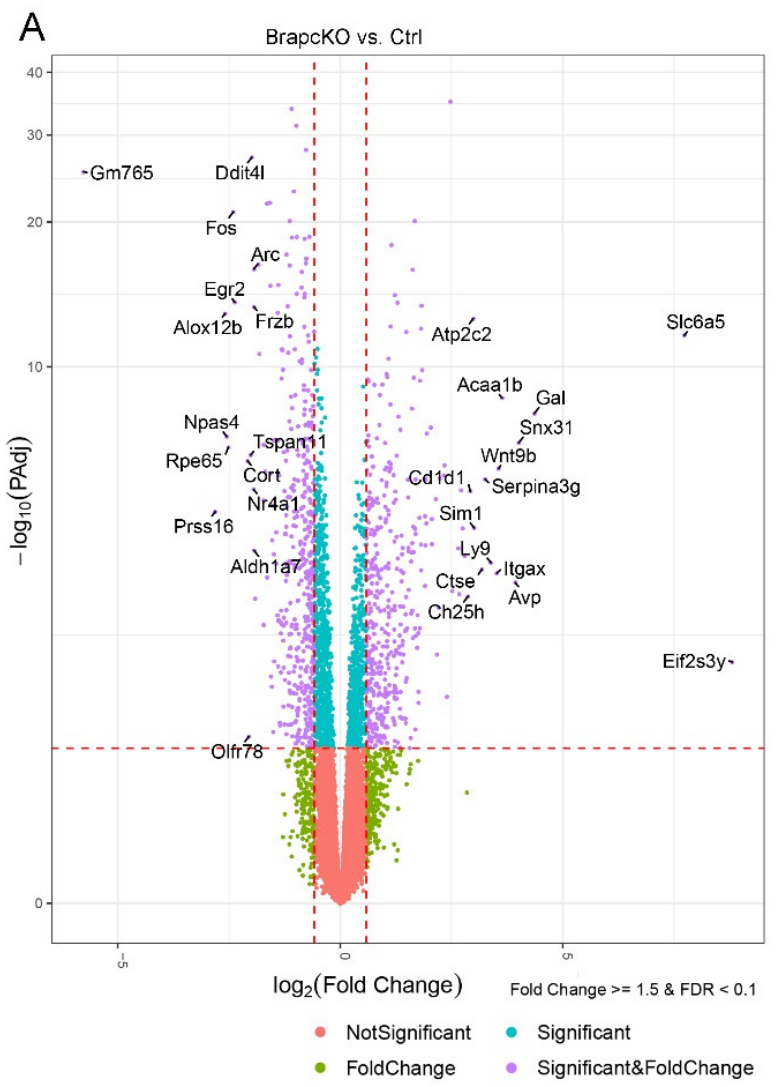

B

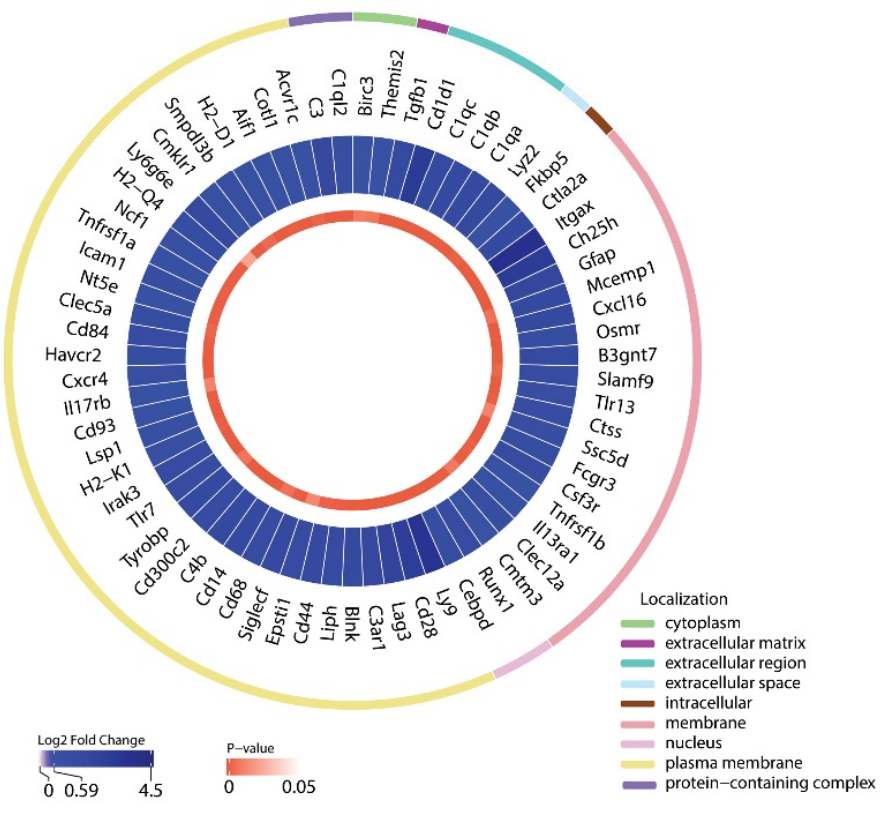

C

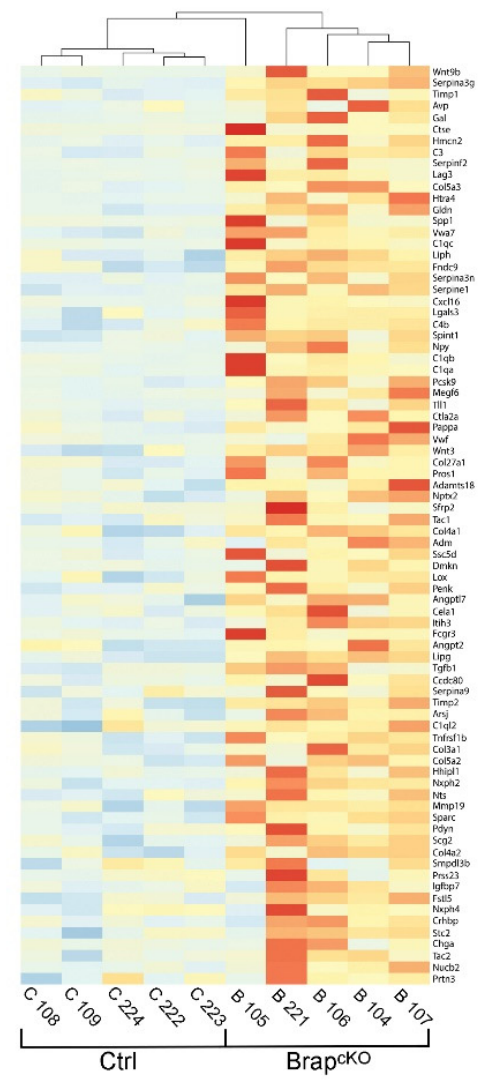

$\mathrm{E}$
D Plasma membrane/ECM

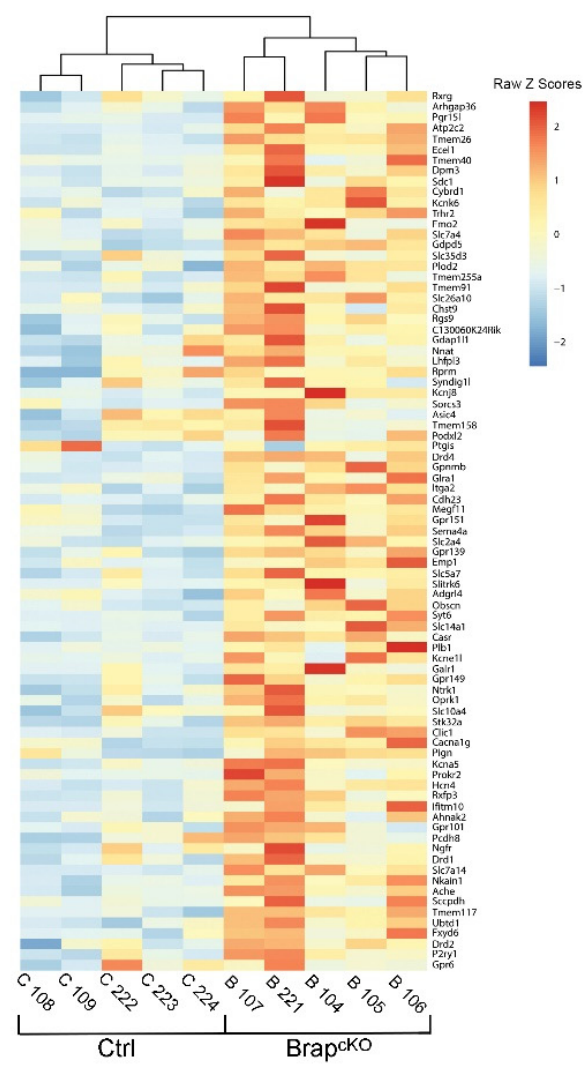

Top 50 Down-regulated Genes

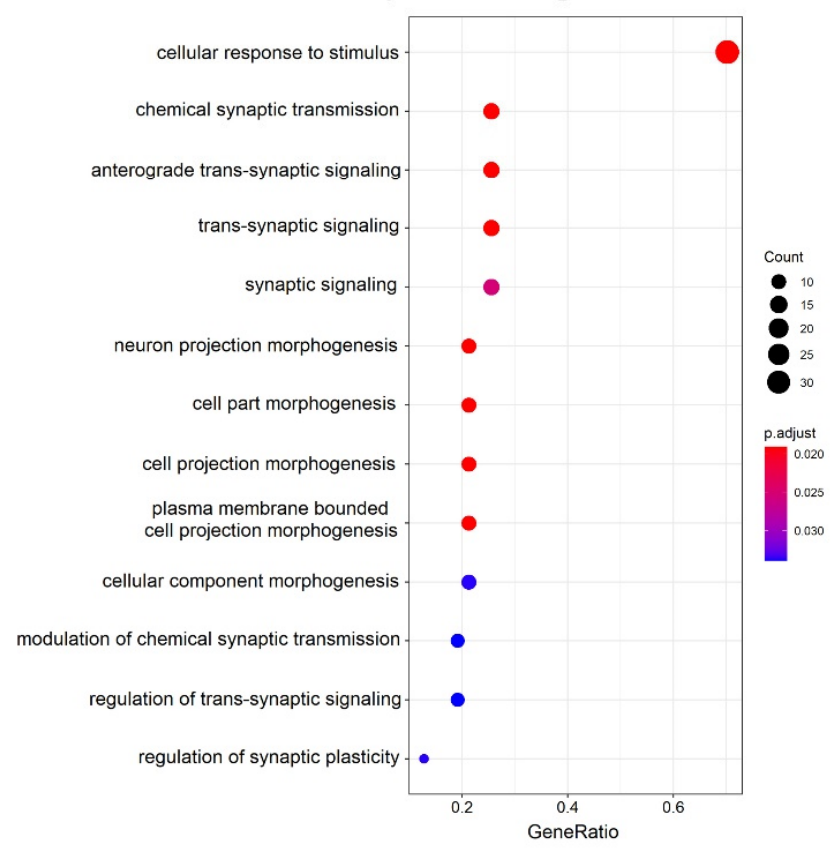




\section{FIGURE 3. DIFFERENTIAL GENE EXPRESSION CAUSED BY BRAP LOF REVEALS INCREASED IMMUNE ACTIVITIES, SASP PROFILES, AND COMPROMISED SYNAPTIC FUNCTION IN CEREBRAL CORTICAL TISSUE.}

A. Volcano plot shows differentially expressed genes (DEGs) between Brap ${ }^{\text {cKONPC }}$ and control cortical tissues.

B. Circos plot presentation of upregulated genes associated with immune cells and innate immunity regulations in Brap ${ }^{\text {cKONPC }}$ relative to control cerebral cortices.

C. Heatmap shows increased expression of genes encoding secreted molecules in Brap ${ }^{\text {cKONPC }}$ relative to control cerebral cortices.

D. Heatmap shows increased expression of genes encoding plasma membrane and extracellular matrix molecules in Brap ${ }^{\mathrm{cKONPC}}$ relative to control cerebral cortices.

E. Gene ontology (GO) enrichment analysis of the top 50 down-regulated genes (by adjusted p-value) in Brap ${ }^{\text {cKONPC }}$ relative to control cortical tissues. Shown are dot plots of biological processes affected by Brap LOF. Note that synaptic signaling, transmission, and cellular response are significantly compromised in Brap ${ }^{\text {cKONPC }}$ cortices. 
bioRxiv preprint doi: https://doi.org/10.1101/2020.10.22.341784; this version posted January 9, 2021. The copyright holder for this preprint (which was not certified by peer review) is the author/funder. All rights reserved. No reuse allowed without permission.

A

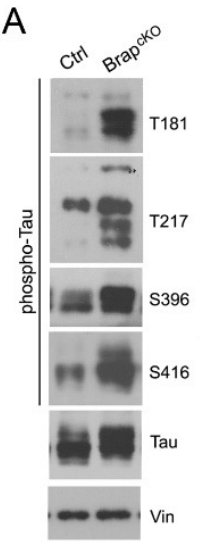

B

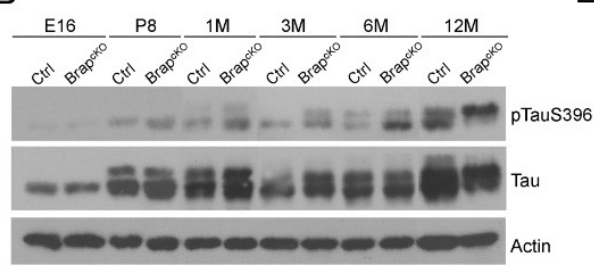

C

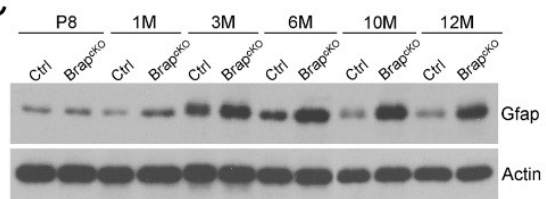

E
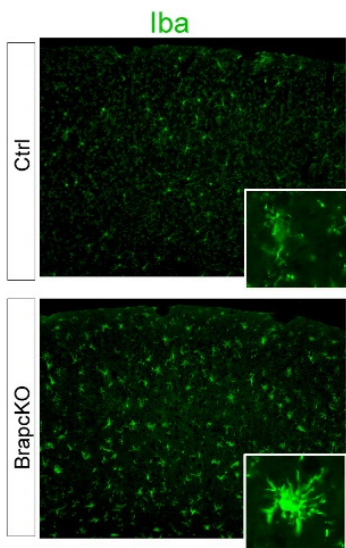
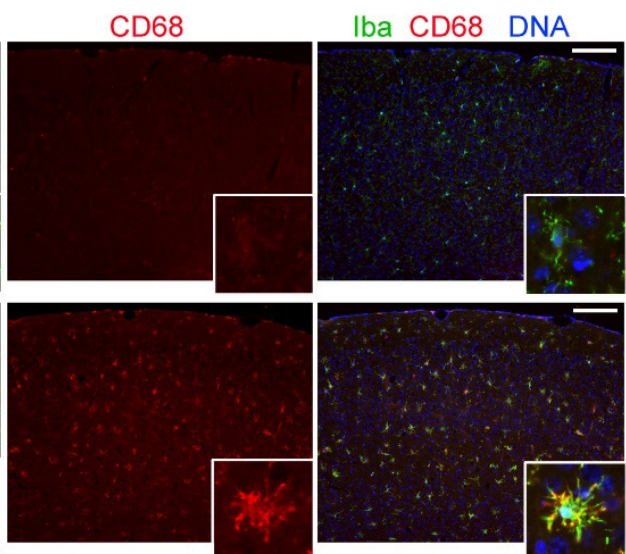

Iba CD68 DNA

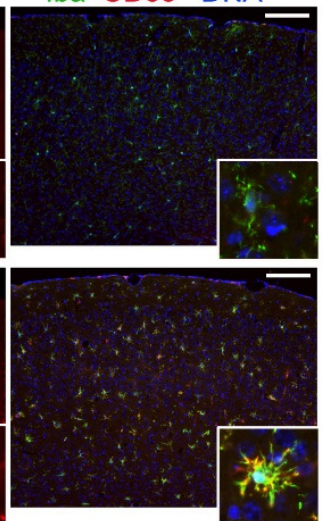

$\mathrm{D}$
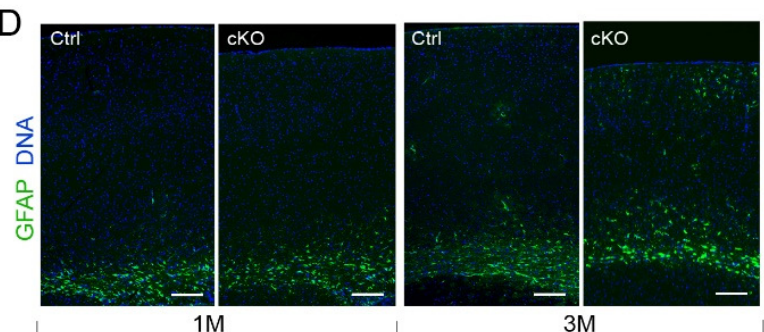

$3 \mathrm{M}$
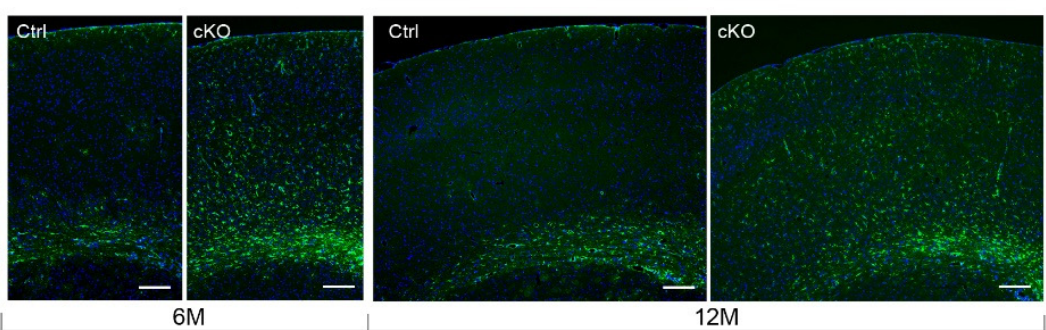

F

G

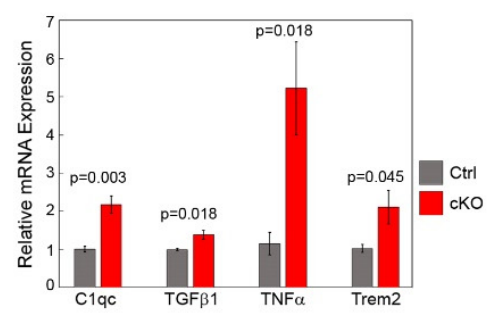

$2 \mathrm{M}$

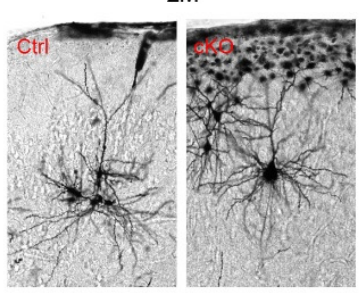

(1)

Ant

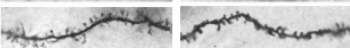

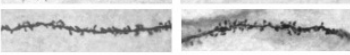

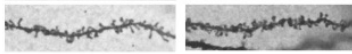

$6 \mathrm{M}$
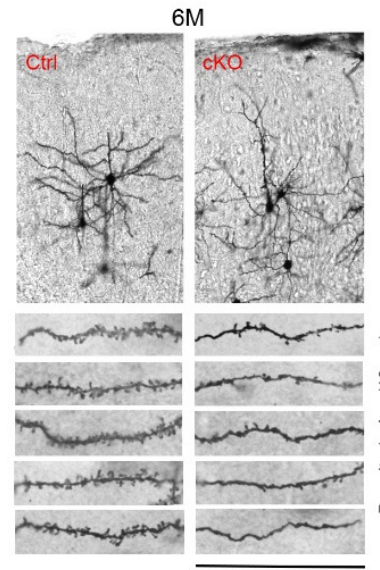

$\mathrm{H}$
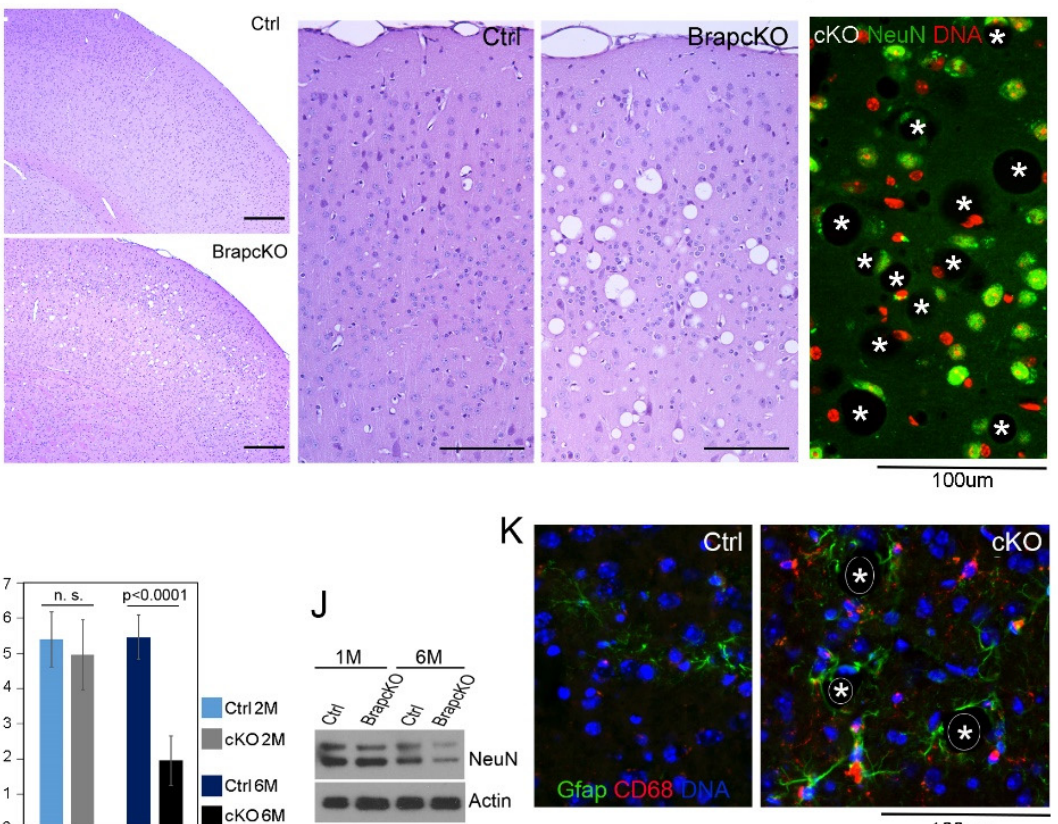

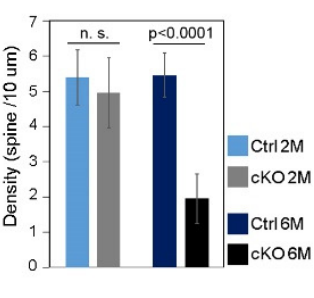




\section{FIGURE 4. BRAP LOF IN CEREBRAL CORTICAL NPCS RESULTS IN NEUROINFLAMMATION AND ACCELERATED NEURODEGENERATION.}

A. Immunoblotting of cerebral cortical total protein extracts from 3-month old Brap ${ }^{\text {cKONPC }}$ and control mice with various anti-phospho-tau antibodies.

B: Immunoblotting of cortical total proteins extracts shows age-dependent progression of tau hyperphosphorylation (on S396) in Brap ${ }^{\text {cKONPC }}$ cortices.

C, D: Gfap immunohistological and immunoblotting analyses show age-dependent progression of astrogliosis in Brap ${ }^{\text {cKONPC }}$ cortices.

E. Double immunohistological staining with anti-Iba (green) and anti-CD68 (red) antibodies shows marked increase in microglia activation in Brap ${ }^{\text {cKONPC }}$ cortices at 3 months. Representative images are shown.

F. qRT-PCR analyses of selected neuroinflammatory genes in 3-month cortical tissues (Mean $\pm \mathrm{SE}$; $\mathrm{n}=4-8$ biological replicates).

G. Representative images and quantification (Mean \pm SD) of Golgi-cox analyses, showing significantly reduced density of dendritic spines in cortical layer $2 / 3$ pyramidal neurons of Brap ${ }^{\text {cKONPC }}$ mice at 6 months.

H. H\&E stained brain sections of 10 months old Brap ${ }^{\text {cKONPC }}$ and control mice, showing spongiform encephalopathy in Brap ${ }^{\text {cKONPC }}$ cortical gray matter.

I. A representative NeuN immunohistological image shows reduced neuronal density and dystrophic or distorted neurons adjacent to spongiosis (positions of vacuoles are marked by asterisks).

J. Immunoblotting of cortical total protein extracts, demonstrating decreased NeuN in Brap ${ }^{\text {cKONPC }}$ cortices at 6month.

K. Representative Gfap (green) and CD68 (red) double immunohistological images, showing increased astrogliosis and microgliosis surrounding the spongiform vacuoles (positions of vacuoles are marked by asterisks).

Nuclear DNA was stained with Hoechst 33342. Bars: 100 um

See also Figure S1. 
bioRxiv preprint doi: https://doi.org/10.1101/2020.10.22.341784; this version posted January 9, 2021. The copyright holder for this preprint (which was not certified by peer review) is the author/funder. All rights reserved. No reuse allowed without permission.

A

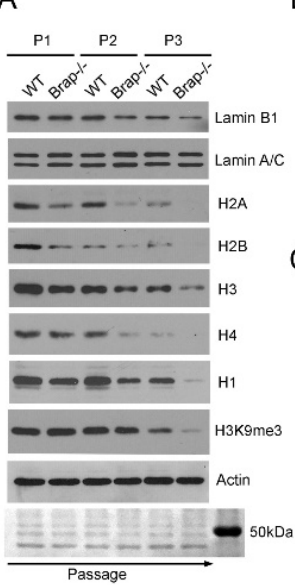

B

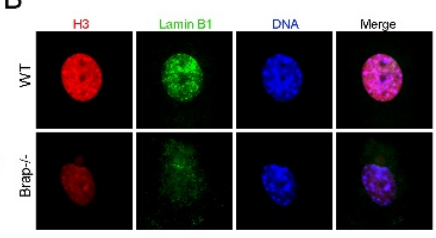

C
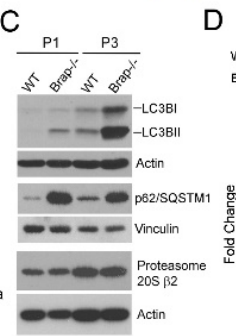

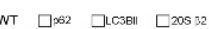
Brap- $-\mathrm{D}_{\mathrm{pO2}}$

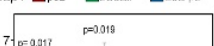

6
5.

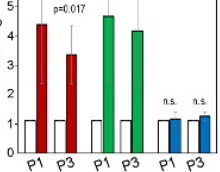

G

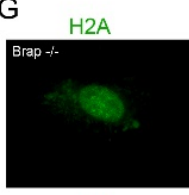

$\mathrm{H} 3$

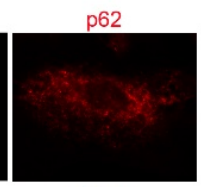

LC3B
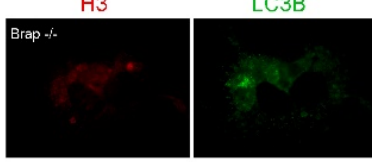

DNA

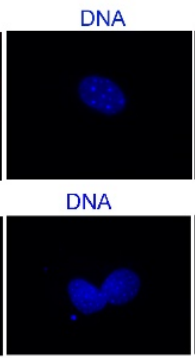

$\mathrm{H}$
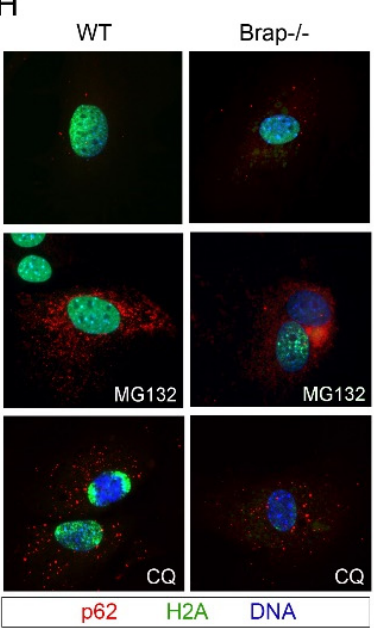

$C Q$

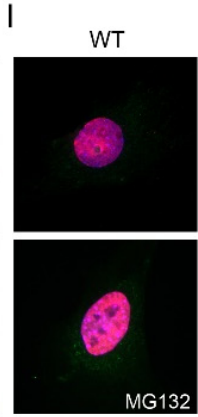

Brap-/-

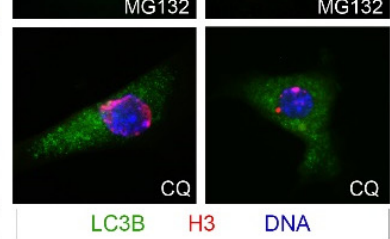

E
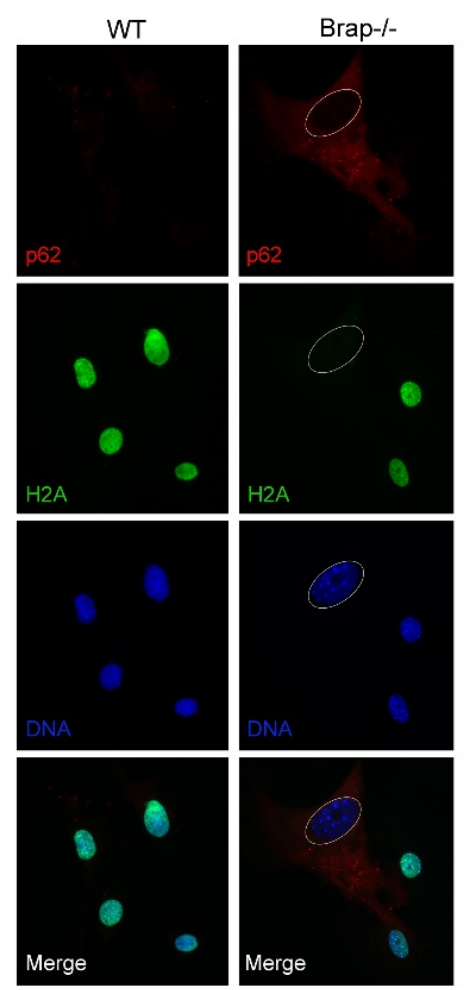

$J$ $\mathrm{MG} 132_{\mathrm{CQ}}= \pm+-\overline{+}$

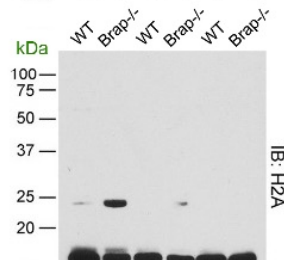

$15-900000$

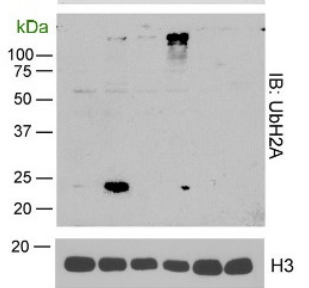

Brap-/-

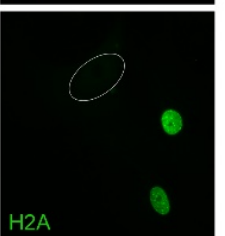

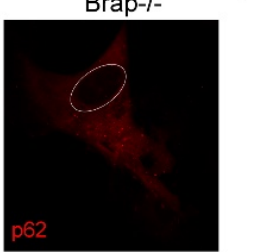

WT
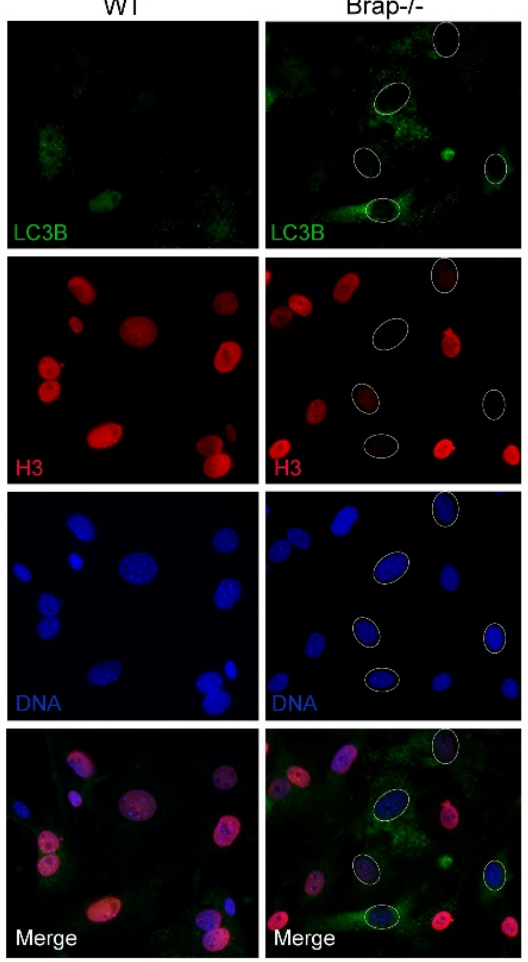

Merge

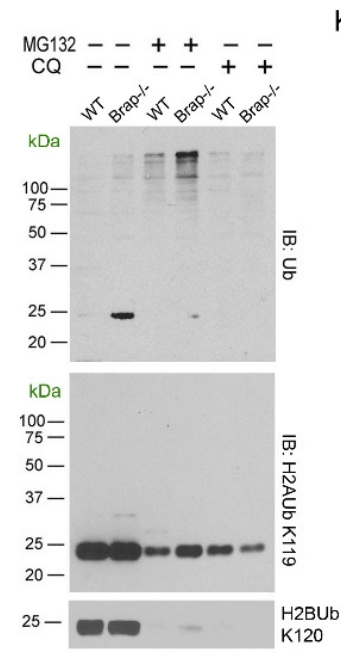

Brap-/-
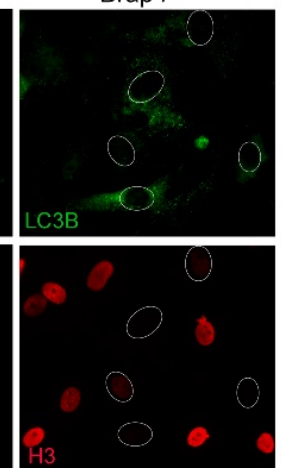

K

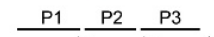

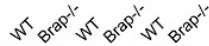
Ka $250-$ $150-$

$100-$

$75-$

$50-$

$37-$

$25-$

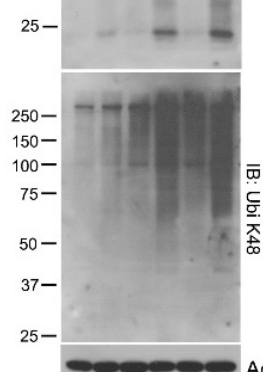




\section{FIGURE 5. CELLULAR SENESCENCE IN BRAP-/- MEFS WAS ASSOCIATED WITH HISTONE H2A UBIQUITINATION AND UPS-MEDIATED PROTEOLYSIS.}

A. Immunoblotting analyses of MEF total protein extracts, showing progressive reduction of Lamin B1 and core histones with increased culture senescence.

B. Representative immunofluorescence images of histone H3 (red) and Lamin B1 (green) double stained MEFs at P3, showing the presence of cytoplasmic Lamin B1 and histone in Brap ${ }^{-/-} \mathrm{MEFs}$.

C. Immunoblotting analyses of autophagy markers LC3B and p62, as well as 20S proteasome catalytic $\beta 2$ subunit, showing increased autophagy flux without alteration of proteasome abundance in Brap ${ }^{-/}$relative to WT MEFs at both $\mathrm{P} 1$ and $\mathrm{P} 3$.

D. Quantification (Mean \pm SD) of p62, LC3BII, and 20S $\beta 2$ levels in WT and Brap ${ }^{-/}$MEFs at P1 and P3, respectively. $n=3-5$ biological replicates.

E, F, G. Representative immunofluorescence images of WT and Brap ${ }^{-/}$MEFs at P3. Antibodies against p62 (red) or LC3B (green) were used to identify autophagosome and co-stained with anti-histone H2A (green) or anti-histone H3 (red). Note that in Brap-/- MEFs, the reduction in nuclear histones coincides with cytoplasmic histone extrusion and correlates with increased autophagy activities but there is little cytoplasmic histoneautophagosome co-localization.

H, I. Representative immunofluorescence images of MEFs treated with UPS blocker MG132 or lysosome blocker chloroquine (CQ), showing that cytoplasmic H2A-p62 or H3-LC3B co-localization is enhanced by MG132 but not by CQ.

J. Immunoblotting analyses of histone extracts from MEFs at P2, showing Brap LOF caused elevated histone $\mathrm{H} 2 \mathrm{~A}$ mono- and poly-ubiquitination. The level of poly-H2Aub was further increased by MG132 but not by CQ, indicating UPS-mediated histone $\mathrm{H} 2 \mathrm{~A}$ proteolysis.

$\mathrm{K}$ : Immunoblotting analyses of MEFs total protein extracts, demonstrating increased levels of polyubiquitinated proteins in senescent Brap ${ }^{-/-}$MEFs.

Nuclear DNA was stained with Hoechst 33342.

See also Figure S2. 


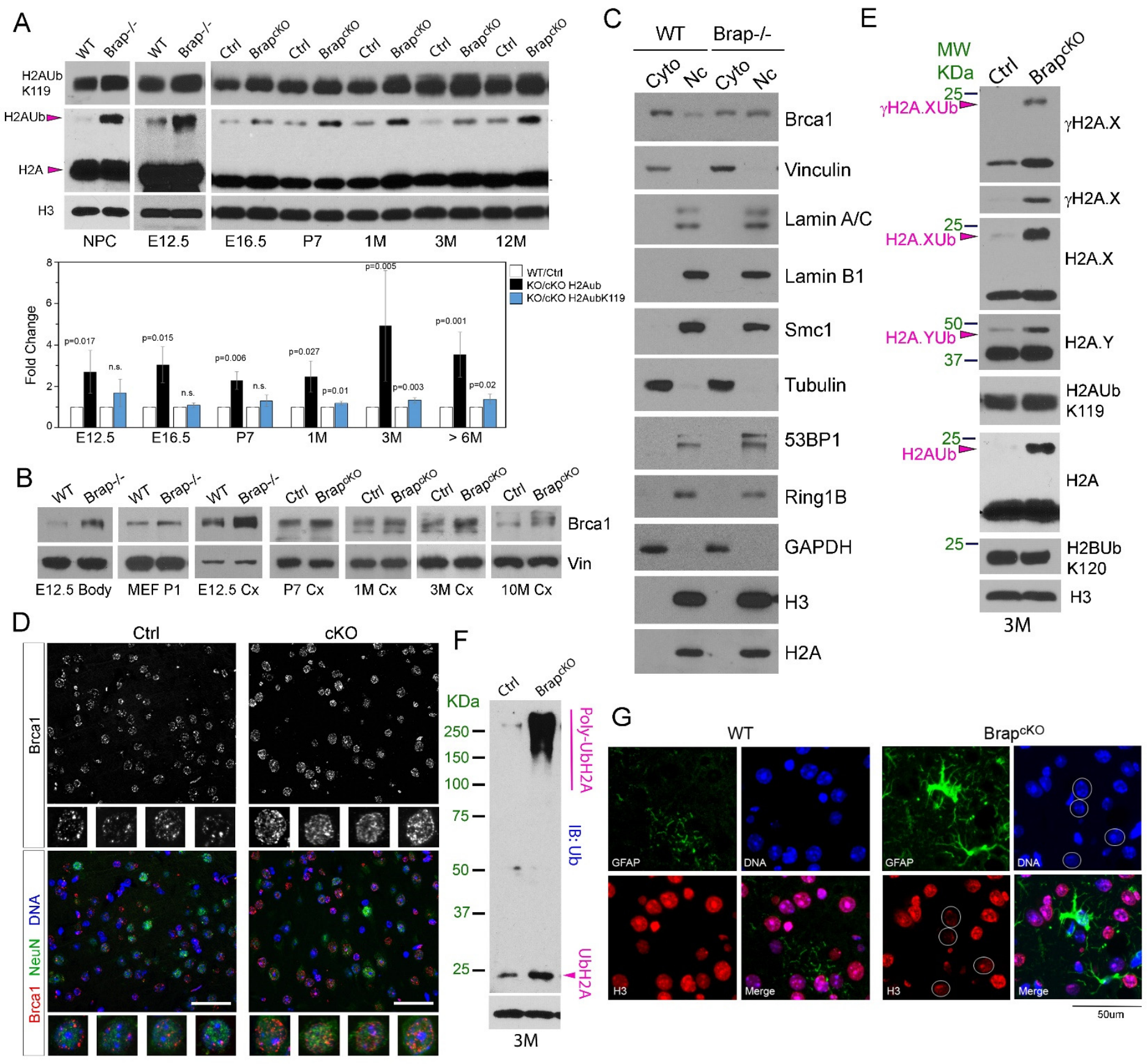




\section{FIGURE 6. HISTONE H2A UBIQUITINATION ACCOMPANIED BY BRCA1 ACTIVATION IS THE HALLMARK PHENOTYPE OF BRAP LOF}

A. Immunoblotting of histone extracts from NPCs as well as from embryonic, neonatal, adult cerebral cortical tissues, and quantification (Mean \pm SD) of increases in histone H2Aub (total H2Aub and H2AubK119, respectively) resulted from Brap LOF. $n=3-6$ biological replicates.

B. Immunoblotting of Brcal in various cells and tissues, showing that Brap LOF results in increased Brca1 abundance.

C. Immunoblotting of nuclear vs. cytoplasmic fractions of MEFs at P1, showing increased nuclear localization of Brcal in $\mathrm{Brap}^{-/-}$cells.

D. Brcal (red) and NeuN (green) double immunohistology images of cerebral cortical sections of Brap ${ }^{\text {cKONPC }}$ and control mice at 4 months of age. Representative images are shown. Note the increased intensity and density of Brcal puncta in the nuclei of Brap ${ }^{\text {cKONPC }}$ cortical neurons $(\mathrm{NeuN}+)$.

E, F. Immunoblotting analyses of histone extracts from cerebral cortical tissues of 3-month old mice, showing increased ubiquitination of $\mathrm{H} 2 \mathrm{~A}$ variants targeted by Brca1 (E) along with total histone $\mathrm{H} 2 \mathrm{~A}$ ubiquitination (F). G. Double immunohistology staining of cortical sections of 4-month old WT or Brap ${ }^{\text {cKONPC }}$ mice with antibodies against Gfap (green) and histone H3 (red), showing reduced nuclear histones in cells surrounded by reactive astrocytes (circles) in Brap ${ }^{\text {cKONPC }}$ cortical tissues. Representative images are shown.

Nuclear DNA was stained with Hoechst 33342. Bars: 50 um.

See also Figure S3. 
bioRxiv preprint doi: https://doi.org/10.1101/2020.10.22.341784; this version posted January 9, 2021. The copyright holder for this preprint (which was not certified by peer review) is the author/funder. All rights reserved. No reuse allowed without permission.
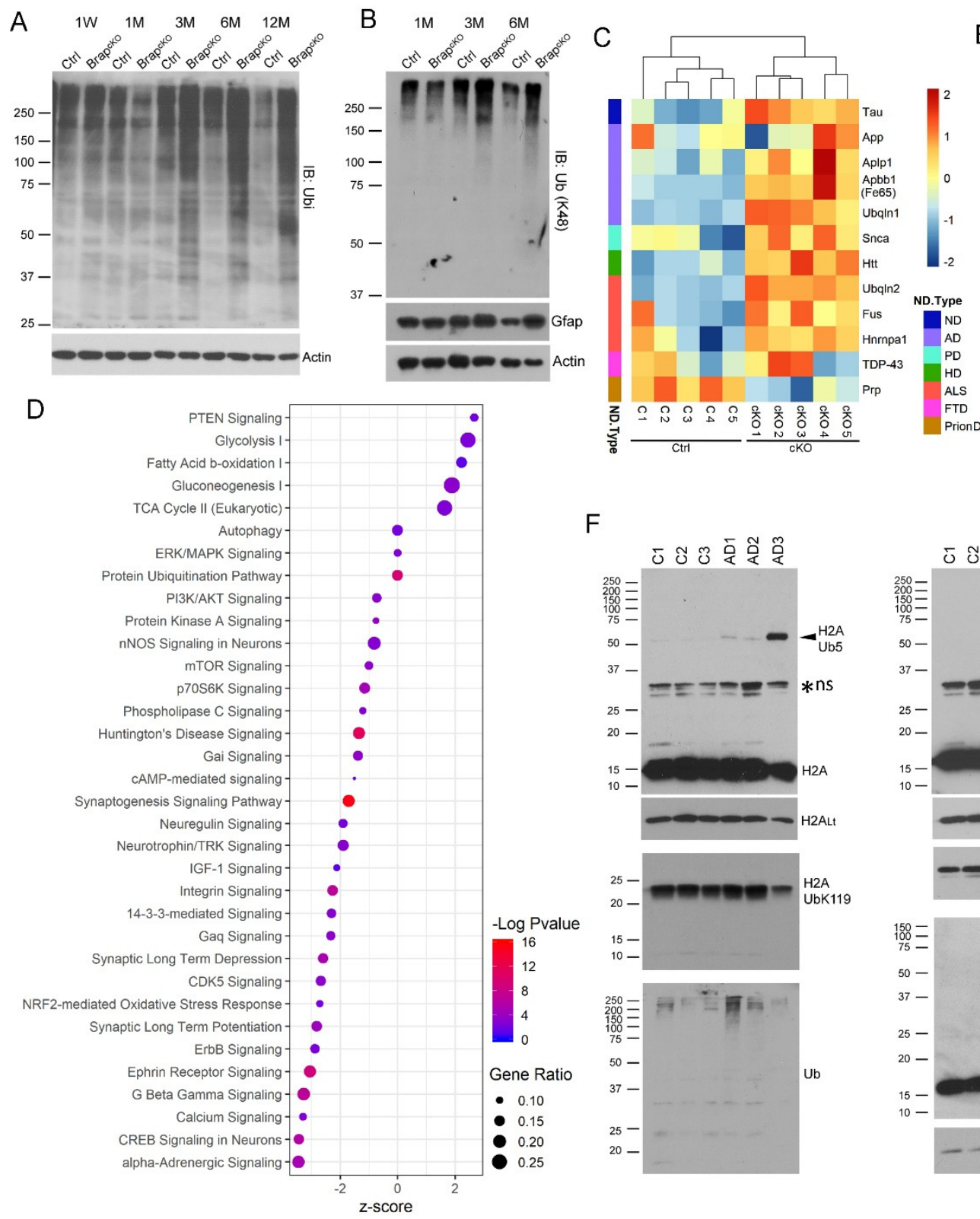

E

F

ธ 0 ช

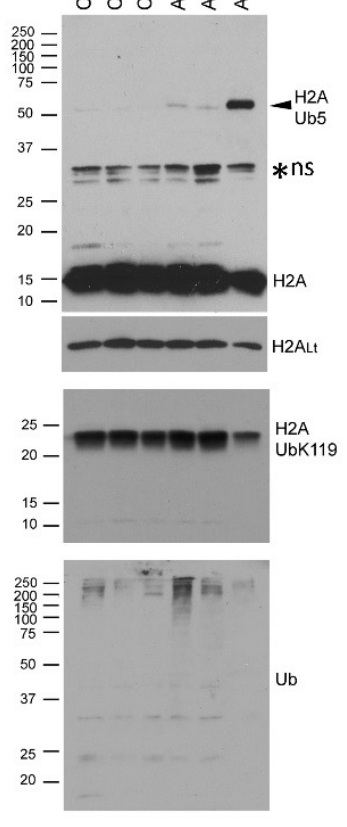

ธ ช ํํㅇำ
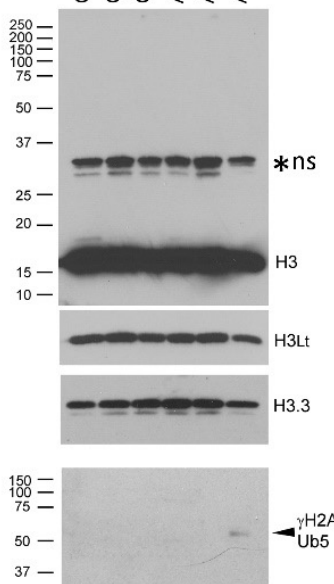

$\gamma$ H2A.X
ธ

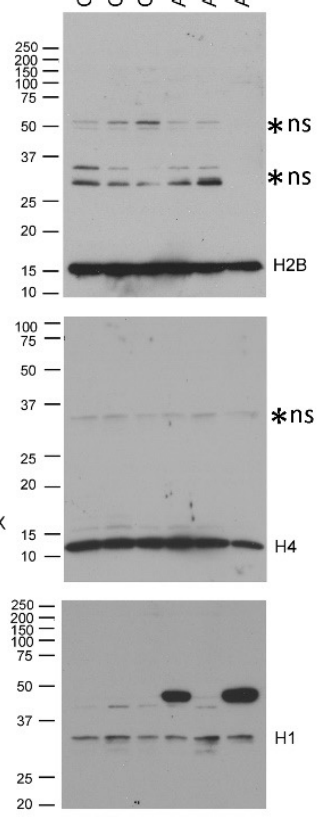




\section{FIGURE 7. IMPAIRED PROTEOSTASIS RESULTING FROM BRAP LOF IN CEREBRAL CORTICAL TISSUE OVERLAPS WITH PROTEOPATHY OF NEURODEGENERATIVE DISORDERS.}

A, B. Ubiquitin immunoblots (total ubiquitination or K48-linked polyubiquitination) of cortical tissue total protein extracts from Brap ${ }^{\mathrm{cKONPC}}$ and littermate control mice, demonstrating age-dependent accumulation of poly-ubiquitinated proteins in the mutant cortical tissues.

C. Heatmap shows selected proteins accumulated in Brap ${ }^{\text {cKONPC }}$ cortical tissue that are also implicated in proteopathy of human neurodegenerative disorders.

D. Dot plot of signaling pathways altered by proteopathy resulted from Brap LOF. Shown were results of IPA of significantly altered proteins revealed by TMT analysis of Brap ${ }^{\text {cKONPC }}$ vs. control cortical proteome.

E. Box and whisker plots of selected results of the TMT analysis, showing significant elevation of multiple histone $\mathrm{H} 2 \mathrm{~A}$ variants in Brap ${ }^{\text {cKONPC }}$ relative to control cortical tissues.

F. Immunoblotting of histone extracts from postmortem cortical tissues of AD patients and age matched normal controls, showing significant elevation of H2Aub in AD cortical tissues. Asterisks denote non-specific bands (n.s.).

See also Figure S4. 


\section{$\underline{\text { Supplemental Figure S1 }}$}

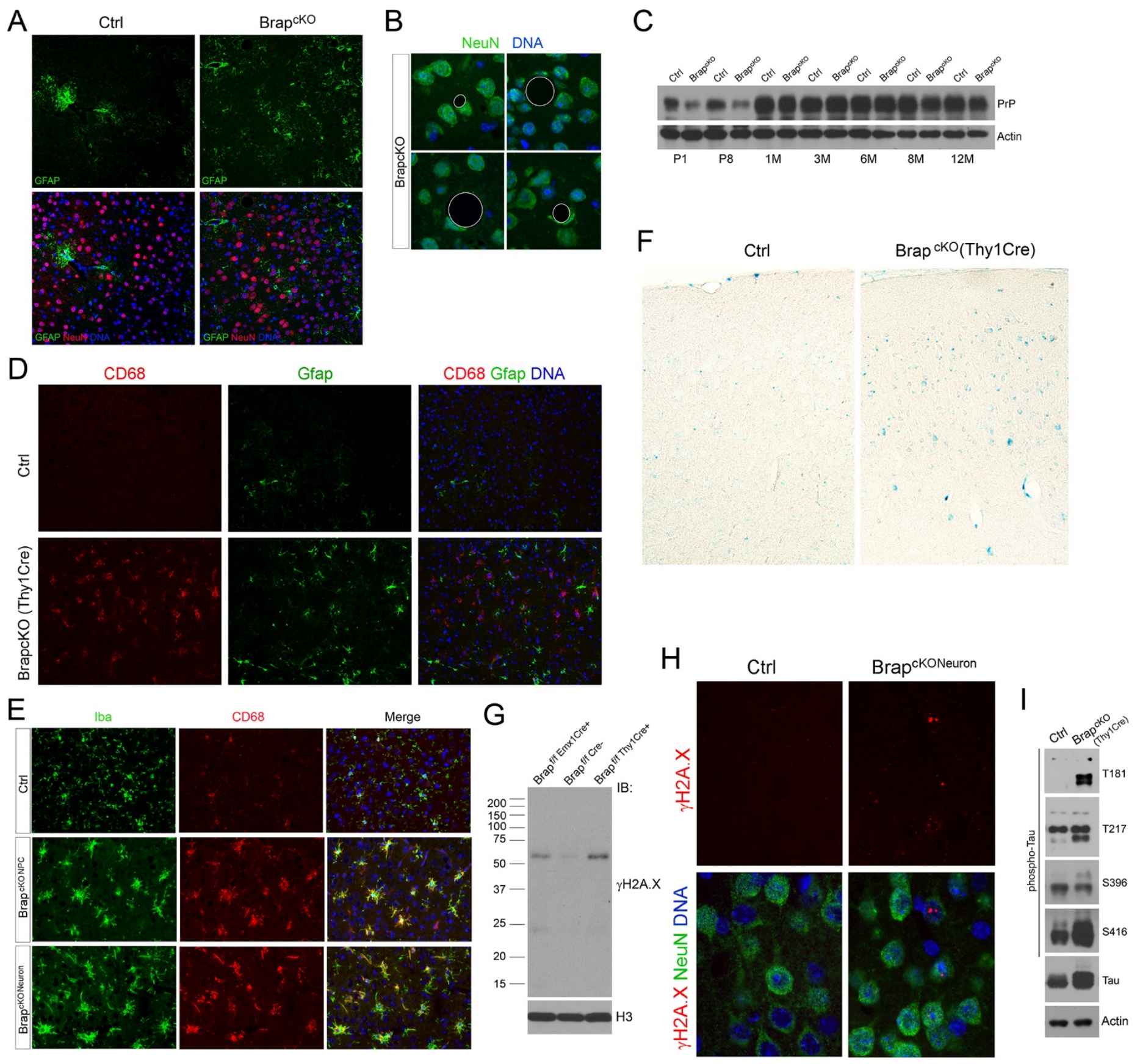




\section{FIGURE S1 (RELATED TO FIGURE 4). PHENOCOPY OF BRAPCKONEURON WITH BRAPCKONPC MICE}

A. NeuN (red) and Gfap (green) double immunohistology (IH) staining of cerebral cortical sections of Brap ${ }^{\text {CKONPC }}$ and control mice at 6 months of age. Representative images are shown.

B. Representative images of anti-NeuN (green) immunohistological staining of cerebral cortical sections from Brap ${ }^{\text {cKONPC }}$ mice at 10 month of age, showing deformed neurons adjacent to spongiform vacuoles (marked by circles).

C. Immunoblotting cortical total protein extract with prion protein (Prp) antibodies of Brap ${ }^{\text {cKONPC }}$ and control mice at various ages.

D. Representative CD68 (red) and Gfap (green) double immunohistological images, showing microgliosis and astrogliosis in the cortical tissue of Brap ${ }^{\text {cKONeuron }}$ mice at 3 months of age.

E. Representative Iba (green) and CD68 (red) double immunohistological images, showing the phenocopy of Brap $^{\text {cKONeuron }}$ and Brap ${ }^{\text {CKONPC }}$ mice with respect to microglia activation in the cerebral cortical tissue at 3 months of age.

F. Senescence-associated $\beta-$ gal analysis of cerebral cortices of Brap ${ }^{\text {cKONeuron }}$ and control mice at 3 months of age. Representative images are shown.

G. Immunoblotting of cerebral cortical histone extracts, showing the phenocopy of Brap ${ }^{\text {cKONeuron }}$ and Brap $^{\text {cKONPC }}$ mice with respect to the elevation of ubiquityl- $\gamma \mathrm{H} 2 \mathrm{~A} . \mathrm{X}$.

H. NeuN (green) and $\gamma$ H2A.X (red) double immunohistological images of cortical section of Brap ${ }^{\text {cKONeuron }}$ and control mice at 3 months of age, showing the presence of neuronal-specific DSBs in Brap ${ }^{\text {cKONeuron }}$ mice.

I. Immunoblotting of cerebral cortical total protein extracts of 3-month old Brap ${ }^{\text {cKONeuron }}$ and control mice with anti-phospho-tau antibodies, showing tau hyper-phosphorylation on multiple residues in mutant cortices.

Nuclear DNA was stained with Hoechst 33342. 


\section{Supplemental Figure S2}

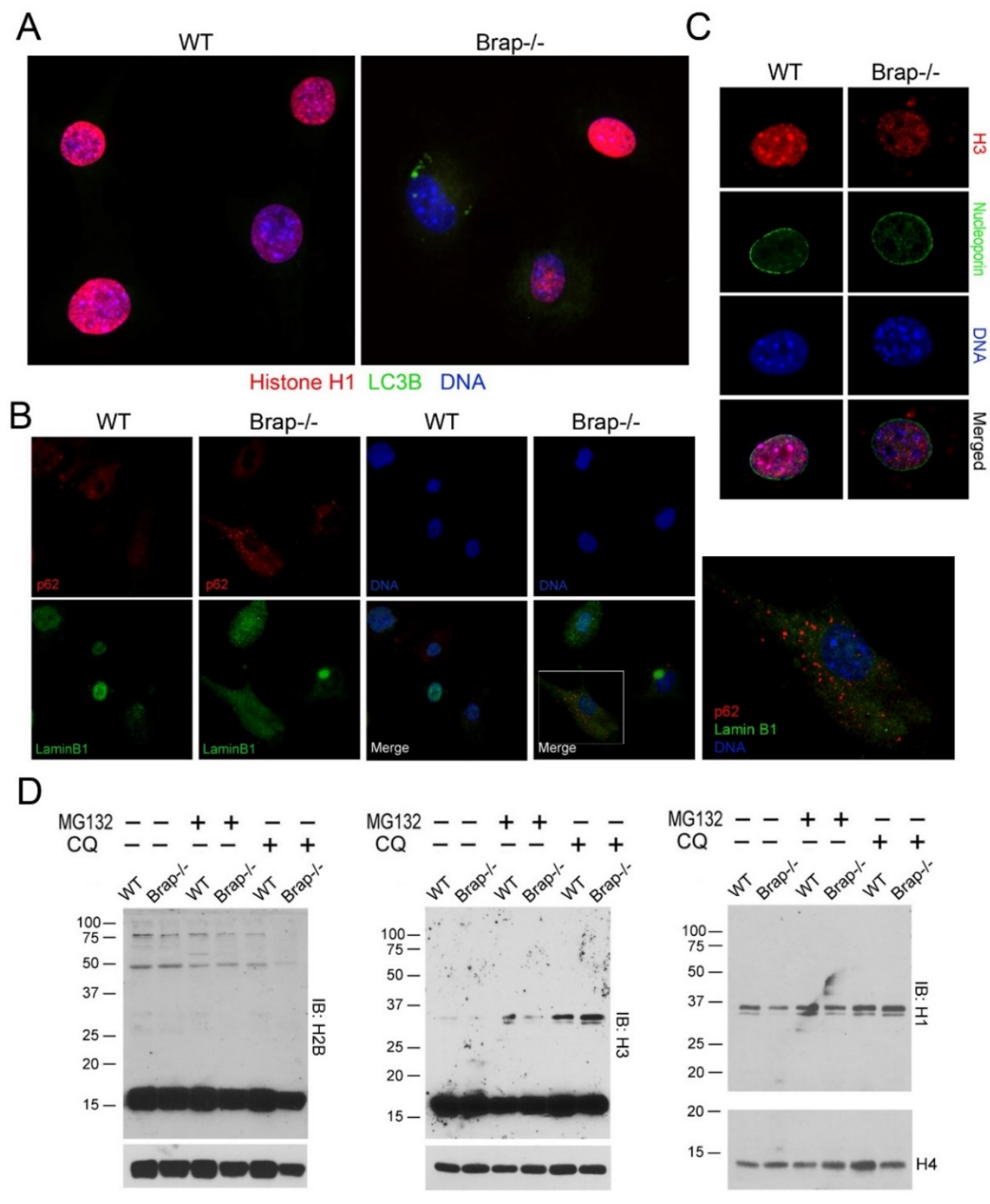

\section{FIGURE S2 (RELATED TO FIGURE 5). BRAP LOF RESULTS IN LOSS OF NUCLEAR HISTONES AND NUCLEAR LAMIN B1.}

A. Immunofluorescence images of WT and Brap ${ }^{-/-} \mathrm{MEF}$ s at P3 stained with antibodies to histone $\mathrm{H} 1$ and antiLC3B, showing the correlation of reduced histone $\mathrm{H} 1$ with increased cytoplasmic LC3B.

B. Immunofluorescence images of WT and Brap ${ }^{-/-}$MEFs at P3 stained with antibodies against Lamin B1 and p62, showing the correlation of reduced nuclear Lamin B1 with increased cytoplasmic Lamin B1 and p62.

C. Immunofluorescence images of WT and Brap ${ }^{-/-}$MEFs at P3 stained with anti-nuceloporin and histone H3, showing intact nuclear envelope despite the presence of cytoplasmic histone H3 in Brap ${ }^{-/-}$MEFs.

D. Immunoblotting analyses of histone extracts from MEFs at P2, showing that the ubiquitination of histone H2B, H3, H4, and H1 was not obviously altered by Brap LOF. 


\section{$\underline{\text { Supplemental Figure S3 }}$}

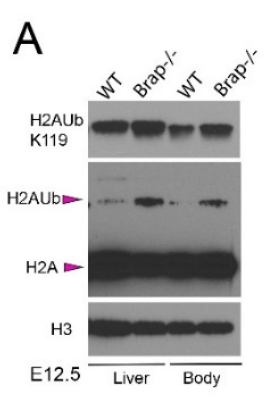

B
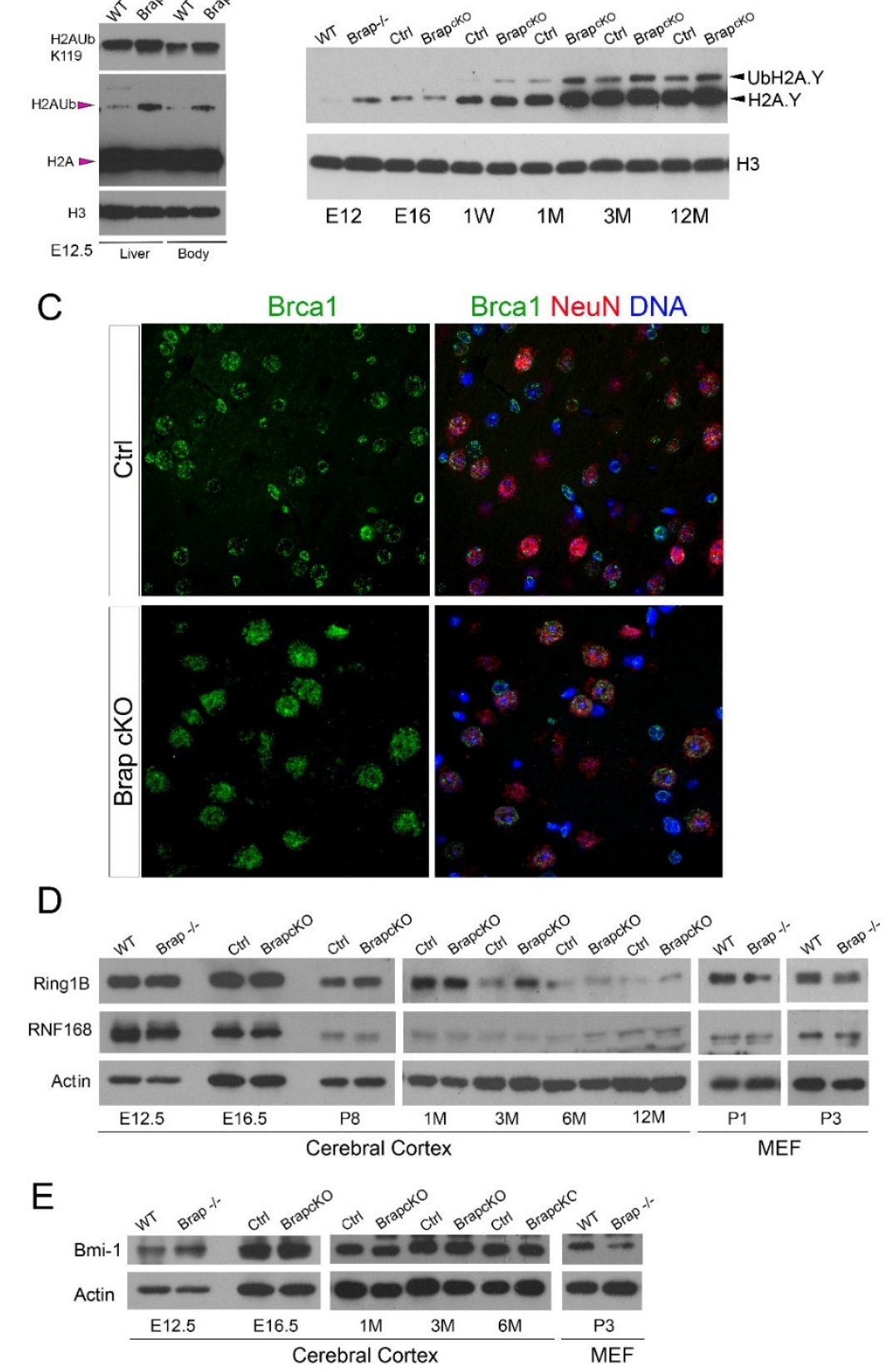

\section{FIGURE S3 (RELATED TO FIGURE 6). BRAP LOF INCREASES HISTONE H2AUB ALONG WITH UBIQUITYL-H2A.Y TARGETED BY BRCA1 E3 LIGASE.}

A. Immunoblotting of histone extracts from embryonic liver and body tissues, showing higher level of $\mathrm{H} 2 \mathrm{Aub}$ in $\mathrm{Brap}^{-/-}$than in of WT mice.

B. Immunoblotting of histone extracts from cerebral cortical tissue at various ages, showing increased H2A.Y (MacroH2A) ubiquitination in postnatal, adult, and aged Brap ${ }^{\mathrm{cKONPC}}$ mice.

C. Brcal (red) and NeuN (green) double immunohistology images of cerebral cortical sections of Brap ${ }^{\mathrm{CKONPC}}$ and control mice at 1 month of age. Representative images are shown.

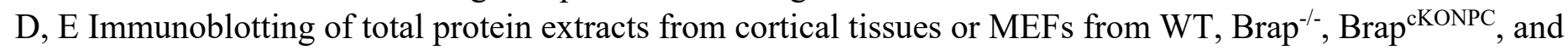
control mice at various ages. 
$\underline{\text { Supplemental Figure S4 }}$
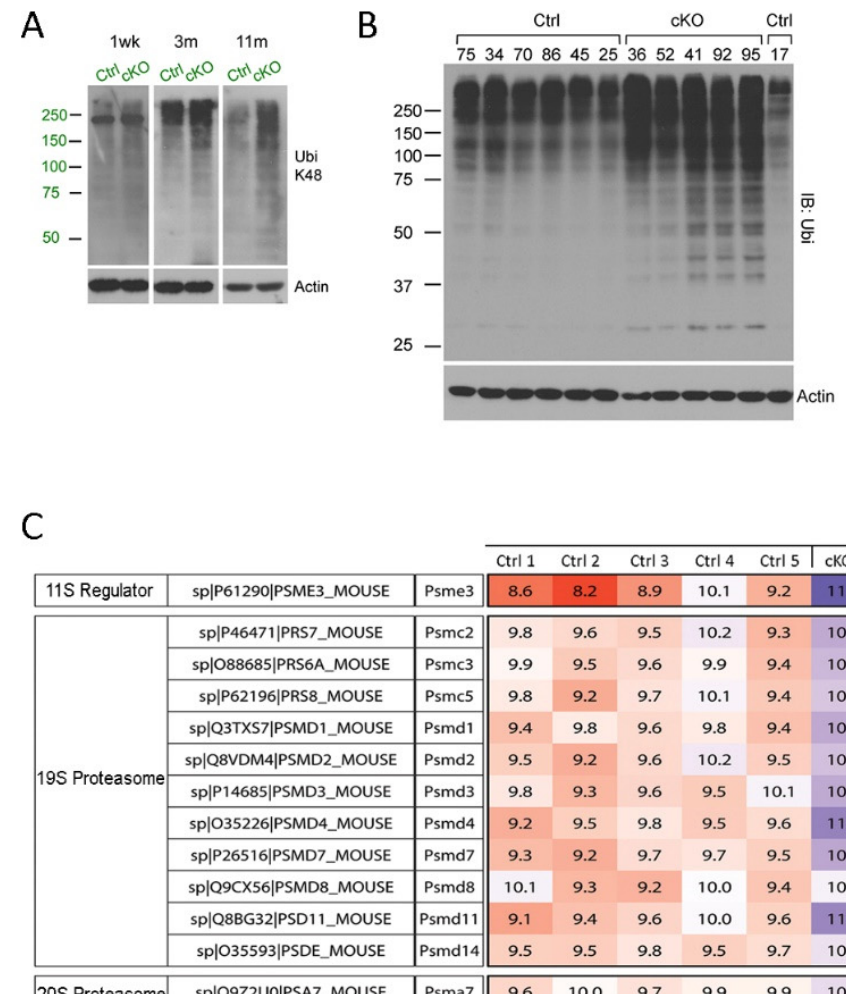

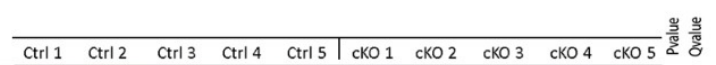

\begin{tabular}{|lccccccccc|c|}
\hline 8.6 & 8.2 & 8.9 & 10.1 & 9.2 & 11.5 & 10.0 & 10.9 & 11.7 & 10.8 \\
\hline
\end{tabular}

\begin{tabular}{|c|c|c|c|c|c|c|c|c|c|}
\hline 8.6 & 8.2 & 8.9 & 10.1 & 9.2 & 11.5 & 10.0 & 10.9 & 11.7 & 10.8 \\
\hline 9.8 & 9.6 & 9.5 & 10.2 & 9.3 & 10.7 & 10.3 & 10.3 & 10.2 & 10.2 \\
\hline 9.9 & 9.5 & 9.6 & 9.9 & 9.4 & 10.6 & 10.3 & 10.2 & 10.5 & 10.1 \\
\hline 9.8 & 9.2 & 9.7 & 10.1 & 9.4 & 10.5 & 10.0 & 10.3 & 10.7 & 10.2 \\
\hline 9.4 & 9.8 & 9.6 & 9.8 & 9.4 & 10.7 & 9.9 & 10.2 & 10.9 & 10.3 \\
\hline 9.5 & 9.2 & 9.6 & 10.2 & 9.5 & 10.5 & 10.4 & 10.0 & 10.8 & 10.2 \\
\hline 9.8 & 9.3 & 9.6 & 9.5 & 10.1 & 10.7 & 10.3 & 10.1 & 10.5 & 10.2 \\
\hline 9.2 & 9.5 & 9.8 & 9.5 & 9.6 & 11.1 & 10.4 & 10.5 & 10.4 & 10.1 \\
\hline 9.3 & 9.2 & 9.7 & 9.7 & 9.5 & 10.7 & 10.4 & 10.6 & 10.5 & 10.3 \\
\hline 10.1 & 9.3 & 9.2 & 10.0 & 9.4 & 10.1 & 10.7 & 10.1 & 10.8 & 10.2 \\
\hline 9.1 & 9.4 & 9.6 & 10.0 & 9.6 & 11.0 & 10.2 & 10.1 & 10.4 & 10.6 \\
\hline 9.5 & 9.5 & 9.8 & 9.5 & 9.7 & 10.2 & 10.4 & 10.4 & 10.5 & 10.5 \\
\hline
\end{tabular}

20S Proteason

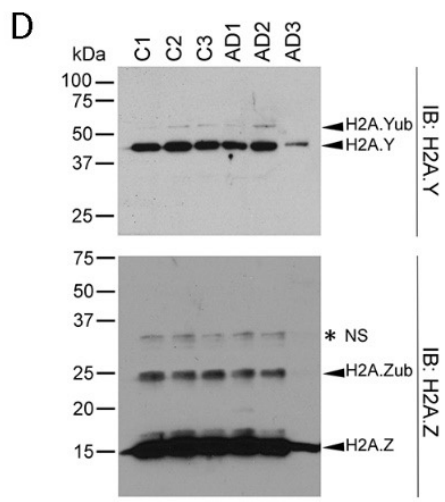

\section{$\frac{20}{20}$} Relative A
$\mathbb{1}_{11}^{12}$
$\mathrm{F}$

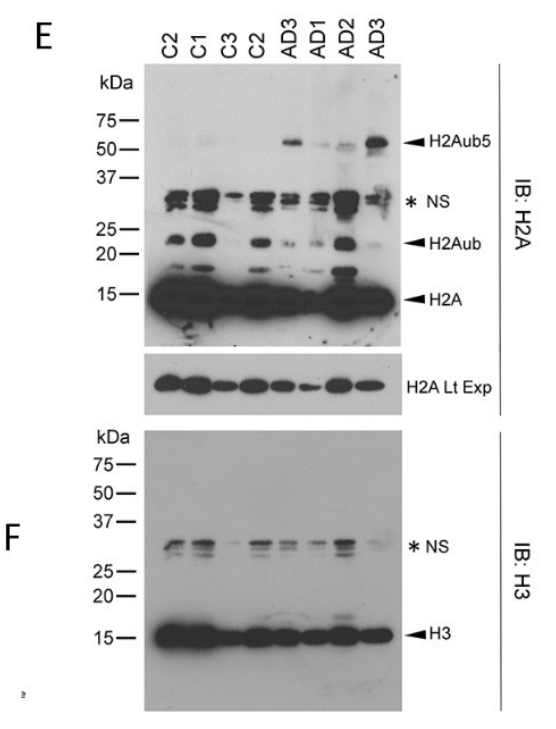

\section{FIGURE S4 (RELATED TO FIGURE 7). ACCELERATED BRAIN AGING OF BRAPC ${ }^{\text {KONPC }}$ MICE IS ASSOCIATED WITH AN ACCUMULATION OF POLY-UBIQUITINATED PROTEINS IN CEREBRAL CORTICAL TISSUES ALONG WITH A COMMON INCREASE OF H2AUB IN BOTH BRAPC ${ }^{K O N P C}$ MICE AND AD PATIENTS.}

A. Immunoblotting of cerebral cortical total protein extracts, showing an age-dependent increase in K48-linked polyubiquitinated proteins that are destined to UPS degradation in Brap ${ }^{\text {cKONPC }}$ cortices.

B. Anti-ubiquitin immunoblotting of total cerebral cortical protein extracts from Brap ${ }^{\text {cKONPC }}$ and control mice at 6 months of age, showing a backlog of polyubiquitinated proteins in all Brap ${ }^{\text {cKONPC }}$ mice.

C. Table and heatmap, showing the significant accumulation of proteasome catalytic and regulatory proteins in Brap ${ }^{\text {cKONPC }}$ cortical tissue revealed by TMT analysis.

D. Immunoblotting AD postmortem cortical tissue histone extracts with antibodies against histone H2A.Y and H2A.Z, respectively. Asterisks denote non-specific bands.

E. Immunoblotting AD postmortem cortical tissue histone extracts with antibodies against histone H2A. This represents a technical replication of Figure 7F. Asterisks denote non-specific bands. 


\section{Graphic Abstract}

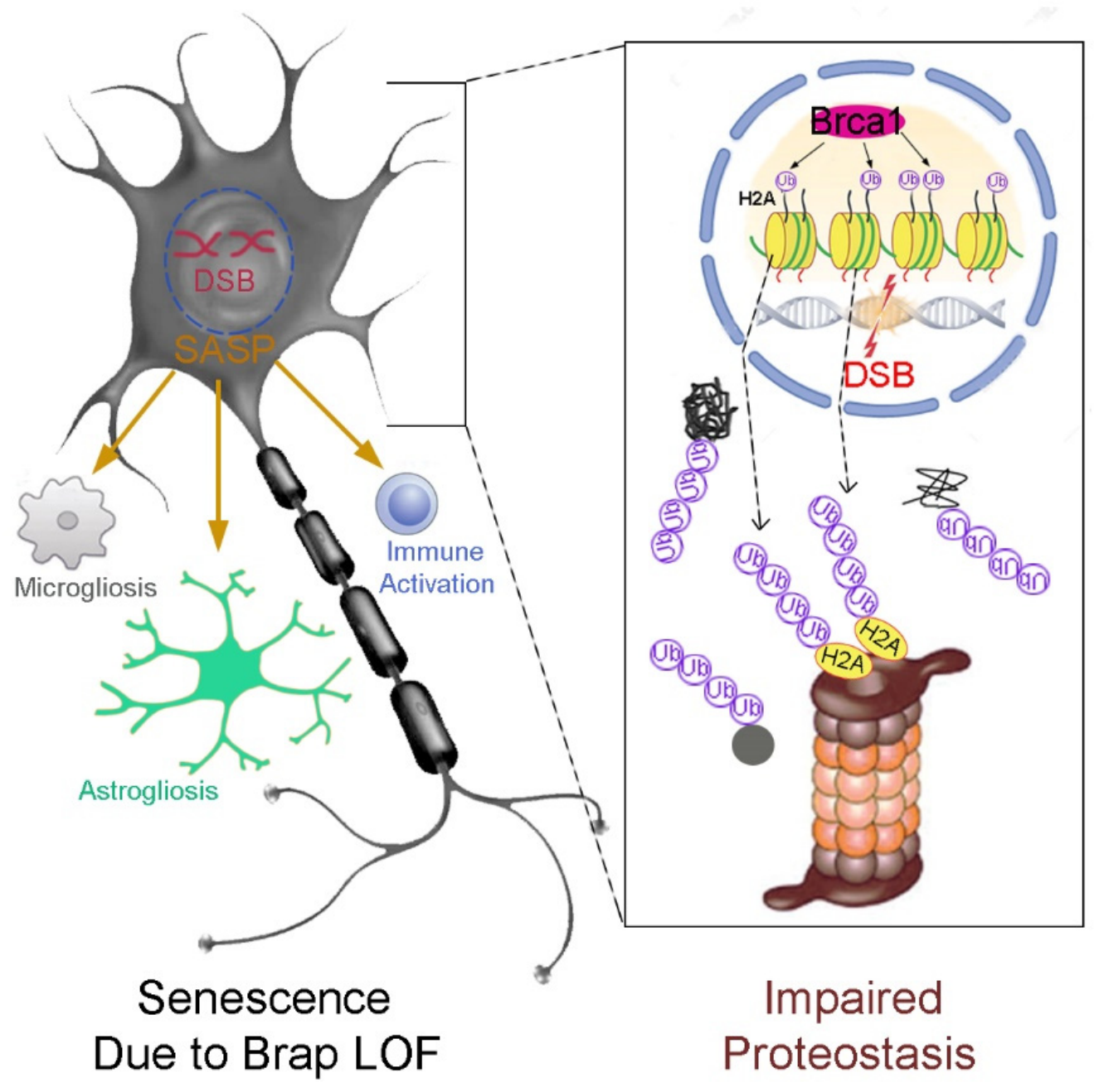

